\title{
UNIFORM A PRIORI ESTIMATES FOR A CLASS OF HORIZONTAL MINIMAL EQUATIONS.
}

\author{
RICARDO SA EARP
}

\begin{abstract}
In the product space $\mathbb{H}^{n} \times \mathbb{R}$, we obtain uniform a priori $C^{0}$ horizontal length estimates, uniform a priori $C^{1}$ boundary gradient estimates, as well as uniform modulus of continuity, for a class of horizontal minimal equations. In two independent variables, we derive a certain uniform global a priori $C^{1}$ estimates and we infer an existence result.
\end{abstract}

\section{INTRODUCTION}

The theory of minimal and constant mean curvature surfaces in the product space $\mathbb{H}^{2} \times \mathbb{R}$, where $\mathbb{H}^{2}$ is the hyperbolic plane is now a rich field of intense research. The notion of vertical graph has a major importance in this theory. The first main results about minimal vertical graphs were derived by B. Nelli and H. Rosenberg [29]. Since then, a significant progress in the theory has been achieved, see, for instance, [36], [22], [23]. When the ambient space is $\mathbb{H}^{n} \times \mathbb{R}$, the notion of vertical mean curvature equation in $n$ independent variables has also been established and developed [40], [38].

On the other hand, there exists also a notion of graph called horizontal graph that has been focused in the theory of minimal and constant mean curvature surfaces [33], [34].

The notion of horizontal graph arises naturally in the theory of hypersurfaces in $\mathbb{H}^{n} \times \mathbb{R}$. There are many interesting examples of minimal and constant mean curvature horizontal graphs in $\mathbb{H}^{n} \times \mathbb{R}$ given by explicit formulas. In fact, there are complete horizontal minimal graphs and there are entire horizontal constant mean curvature graphs [4], [5], [11].

We notice that there is a different notion of horizontal graph called horizontal graph with respect to a geodesic of $\mathbb{H}^{2}$, that has been applied to prove a Schoen type result for minimal surfaces in $\mathbb{H}^{2} \times \mathbb{R}[18]$.

Date: May 20, 2012.

2000 Mathematics Subject Classification. 53C42, 35J25.

The author wish to thank CNPq, FAPERJ of Brazil, for financial support. 
We choose the upper half-plane model of hyperbolic plane $\mathbb{H}^{2}=$ $\{(x, y), y>0\}$, endowed with the hyperbolic metric $d \sigma^{2}=\frac{d x^{2}+d y^{2}}{y^{2}}$. A horizontal graph in $\mathbb{H}^{2} \times \mathbb{R}$ is the set $S=\{(x, g(x, t), t),(x, t) \in$ $\Omega\} \subset \mathbb{H}^{2} \times \mathbb{R}$, where $\Omega \subset \partial_{\infty} \mathbb{H}^{2} \times \mathbb{R}$ is a domain and $g(x, t)>0$, for every $(x, t) \in \Omega$. This means that in any slice of $\mathbb{H}^{2} \times \mathbb{R}$ given by $t=$ cst, each horizontal geodesic $x=$ cst, $y>0$ intersects $S$ in one point at most. We call the positive function $g(x, t)$ the horizontal length of the graph. If $S$ is a horizontal minimal graph in $\mathbb{H}^{2} \times \mathbb{R}$ the positive function $g(x, t)$ satisfies equation (4).

In this paper, we derive uniform a priori horizontal length estimates and uniform a priori boundary gradient estimates for positive smooth solutions of a wider class of quasilinear elliptic equations, indexed by the parameter $\epsilon \in[0,1]$. We call such equations the $\epsilon$-horizontal minimal equations (see equation (5)). If $\epsilon=0$, we find the horizontal minimal equation.

We point out that if $\epsilon>0$, the equation is strictly elliptic, but if $\epsilon=0$ this fact is no longer true in general. Indeed, there are many examples that can be constructed to show lack of strictly ellipticity for the horizontal minimal equation, even on bounded domains. We give now a significant example of this phenomenon in two variables. Just take $g(x, t)=x \sinh t, 0<x<1,0<t<1$ [33, Equation 32]. It is easy to verify that $y=g(x, t)$ satisfies the horizontal minimal equation. A simple verification shows that if $x \rightarrow 0$, then the first eigenvalue of the horizontal minimal operator goes to zero. On the other hand, it is amazing that the vertical minimal equation is strictly elliptic for all values of the independent variables [33, Equation 4], [36, Equation 6].

The uniform $C^{0}$ estimates for positive smooth solutions on bounded domains, continuous up to the boundary, is obtained by comparing, by maximum principle, such solutions with certain geometric subsolutions and supersolutions. These a priori $C^{0}$ estimates depends on the width of the domain and on the boundary value data.

In the same sprit as in [35], we obtain uniform a priori boundary gradient estimates on arbitrary bounded smooth convex domains for solutions of equation (5), that are smooth and positive up to the boundary. Indeed, we provide uniform analytic barriers on bounded $C^{0}$ convex domains to ensure uniform modulus of continuity for positive continuous solutions of (5) that are continuous and positive up to the boundary.

In the case of two variables, we are able to derive uniform a priori global $C^{1}$ estimates on bounded smooth convex domains, making an additional strong assumption on the horizontal length. However, this 
assumption is compatible with the geometry of the ambient space, i.e., it is invariant by hyperbolic translations.

Notice that there is a non-existence result that follows from the asymptotic principle proved by E. Toubiana and the author in [36, Theorem 2.1]. Namely, there is no horizontal minimal graph given by a function $g \in C^{2}(\Omega) \cap C^{0}(\bar{\Omega})$ on a bounded strictly convex domain $\Omega$, taking zero boundary data on $\partial \Omega$. Furthermore, there is no horizontal minimal graph in $\mathbb{H}^{2} \times \mathbb{R}$, over a bounded Jordan domain $\Omega$ strictly contained in an horizontal slab of height $\pi$, given by a function $g \in C^{2}(\Omega) \cap C^{0}(\bar{\Omega})$, taking zero asymptotic boundary data on $\partial \Omega[36$, Corollary 2.1].

We obtain an existence result for the $\epsilon$-horizontal minimal equation in two independent variables on bounded smooth convex domains, taking certain smooth positive boundary value data. This existence result is, in certain sense, a counterpart of the non-existence results stated in the foregoing paragraph. Particularly, we derive the following consequence. Given a smooth positive function $f$ on $\partial \Omega$; if $c_{0}$ is a constant large enough, then there exists a solution of the $\epsilon$ - horizontal minimal equation in $\Omega$, taking boundary value data $f+c_{0}$ on $\partial \Omega$. Actually, it suffices to take $c_{0}$ greater than the sum of the oscillation of the boundary data $f$ with the half of the horizontal width of the domain $\Omega$.

Existence results for the Dirichlet problem for the $\epsilon$-horizontal minimal equation in $n \geqslant 3$ variables is an open problem.

It is worthwhile to notice that the horizontal minimal equation (4) is not invariant by Euclidean translations along the $y$-axis. Of course, the structure of the horizontal minimal equation does not ensure the uniqueness of the solution of the Dirichlet problem on bounded domains. In fact, the model minimal surfaces described in [36, Proposition 2.1] and used in [34, Proof of Theorem 1.1] to prove a Bernstein type theorem, shows that there is a family of horizontal minimal graphs over a rectangle $\mathcal{R}$ of $t$-height greater than $\pi$ taking zero boundary data over $\partial \mathcal{R}$. Furthermore, over domains of arbitrarily large $x$ - width, each element of this family takes arbitrarily small constant boundary data over a bounded domain in the rectangle, but the family attains arbitrarily large horizontal length. Thus, it follows that the dependence of the a priori horizontal length estimates on the width of the domain is quite natural.

However, over certain admissible convex domains such that the boundary data has an admissible bounded slope condition, the solution is the Morrey's solution of the Plateau problem [24]. This result follows from the uniqueness theorem established in [34]. The uniqueness or 
the non-uniqueness of the Dirichlet problem for the $\epsilon$ - horizontal minimal equation on bounded convex domains and positive boundary data is an open problem.

We remark that we need to consider the family of $\epsilon$ - horizontal minimal equations in order to prove the existence of our Dirichlet problem for the horizontal minimal equation. The scheme of our construction is the following. First, we prove the existence result in the strictly elliptic situation, $i$. e. when $\epsilon>0$. Then, we are able to deduce it for $\epsilon=0$; that is, we get a solution for the horizontal minimal equation. In fact, in view of our uniform a priori $C^{1}$ global estimates and elliptic theory, this solution is obtained as the limit in the $C^{2}$-topology of a sequence $g_{\epsilon_{n}}$, as $0<\epsilon_{n} \rightarrow 0$, satisfying the $\epsilon_{n^{-}}$horizontal minimal equation.

ACKNowledgments: The author warmly thanks to Eric Toubiana and Barbara Nelli for their valuable observations.

\section{The $\epsilon$-HORIzOntal minimal EQUATION IN $\mathbb{H}^{n} \times \mathbb{R}$.}

In this section we give some computations, some model subsolutions and supersolutions and we write down some basic properties of the $\epsilon$-horizontal minimal equation in the product space $\mathbb{H}^{n} \times \mathbb{R}$.

We choose the upper half-plane model of the hyperbolic plane $\mathbb{H}^{n}=$ $\left\{\left(x_{1}, x_{2}, \ldots, x_{n-1}, y\right), y>0\right\}$, endowed with the hyperbolic metric $d \sigma^{2}=$ $\frac{d x_{1}^{2}+\cdots+d x_{n-1}^{2}+d y^{2}}{y^{2}}$. A horizontal graph in $\mathbb{H}^{n} \times \mathbb{R}$ is the set $S=$ $\left\{\left(x_{1}, x_{2}, \ldots, x_{n-1}, g\left(x_{1}, x_{2}, \ldots, x_{n-1}, t\right), t\right),\left(x_{1}, x_{2}, \ldots, x_{n-1}, t\right) \in \Omega\right\} \subset$ $\mathbb{H}^{n} \times \mathbb{R}$, where $\Omega \subset \partial_{\infty} \mathbb{H}^{n} \times \mathbb{R}[37]$ is a domain and $g\left(x_{1}, x_{2}, \ldots, x_{n-1}, t\right)>$ $0,\left(x_{1}, x_{2}, \ldots, x_{n-1}, t\right) \in \Omega$. This means that at any slice of $\mathbb{H}^{n} \times \mathbb{R}$ given by $t=$ cst, each horizontal geodesic $\left(x_{1}, x_{2}, \ldots, x_{n-1}\right)=$ cst, $y>0$ intersects $S$ at most on one point.

We give now the computations of some important geometric quantities of the horizontal graph $S$.

Let us define the quantity

$W:=g^{2}\left(1+g_{x_{1}}^{2}+\cdots+g_{x_{n-1}}^{2}\right)+g_{t}^{2}$.

- The coefficients of the first fundamental form are

$$
\begin{aligned}
& g_{k k}=\frac{1+g_{x_{k}}^{2}}{g^{2}}, 1 \leqslant k<n, g_{j k}=\frac{g_{x_{j}} g_{x_{k}}}{g^{2}}, 1 \leqslant j<k<n, \\
& g_{n k}=\frac{g_{x_{k}} g_{t}}{g^{2}}, 1 \leqslant k<n, g_{n n}=\frac{g^{2}+g_{t}^{2}}{g^{2}} .
\end{aligned}
$$

- The inverse of the matrix $\left(g_{i j}\right)$ is the matrix $g^{i j}$ given by

$$
\begin{aligned}
& g^{k k}=\left[g^{2}\left(1+g_{x_{1}}^{2}+\cdots+\widehat{g_{x_{k}}^{2}}+\cdots g_{x_{n-1}}^{2}\right)+g_{t}^{2}\right] \cdot \frac{g^{2}}{W}, \\
& 1 \leqslant k<n .
\end{aligned}
$$




$$
\begin{aligned}
& g^{k j}=-g^{2} g_{x_{j}} g_{x_{k}} \cdot \frac{g^{2}}{W}, 1 \leqslant j<k<n . \\
& g^{n k}=-g_{t} g_{x_{k}} \cdot \frac{g^{2}}{W}, 1 \leqslant k<n . \\
& g^{n n}=\left(1+g_{x_{1}}^{2}+\cdots+g_{x_{n-1}}^{2}\right) \cdot \frac{g^{2}}{W} .
\end{aligned}
$$

Let us assume that our horizontal graph $S$ is oriented by the unit normal $N$ given by

$$
N=\left(-g_{x_{1}} g^{2},-g_{x_{2}} g^{2}, \ldots,-g_{x_{n-1}} g^{2}, g^{2},-g_{t}\right) \cdot \frac{1}{W^{1 / 2}}
$$

- The coefficients of the second fundamental form are given by

$$
\begin{aligned}
b_{k k} & =\left(\frac{1}{g}+g_{x_{k} x_{k}}+\frac{g_{x_{k}}^{2}}{g}\right) \cdot \frac{1}{W^{1 / 2}}, 1 \leqslant k<n . \\
b_{k j} & =\left(g_{x_{k} x_{j}}+\frac{g_{x_{k}} g_{x_{j}}}{g}\right) \cdot \frac{1}{W^{1 / 2}}, 1 \leqslant j<k<n . \\
b_{n k} & =g_{t x_{k}} \cdot \frac{1}{W^{1 / 2}}, 1 \leqslant k<n . \\
b_{n n} & =\left(g_{t t}-\frac{g_{t}^{2}}{g}\right) \cdot \frac{1}{W^{1 / 2}} .
\end{aligned}
$$

Proposition 2.1. Let $S$ be a horizontal graph oriented by the unit normal $N$ given by (1). Let $H=H(N)$ be the mean curvature of $S$.

Then the horizontal mean curvature equation is given by

(2) $\mathcal{M}_{\mathcal{H}}(g)=\frac{n H}{g^{2}}\left(g^{2}\left(1+g_{x_{1}}^{2}+\cdots+g_{x_{n-1}}^{2}\right)+g_{t}^{2}\right)^{3 / 2}$

where

$$
\begin{aligned}
& \mathcal{M}_{\mathcal{H}}(g):=\sum_{k=1}^{n-1} g_{x_{k} x_{k}}\left[g^{2}\left(1+g_{x_{1}}^{2}+\cdots+\widehat{g_{x_{k}}^{2}}+\cdots g_{x_{n-1}}^{2}\right)+g_{t}^{2}\right]+\left(1+\sum_{k=1}^{n-1} g_{x_{k}}^{2}\right) g_{t t} \\
& -2 \sum_{k=1}^{n-1} g_{x_{k}} g_{t} g_{x_{k} t}-2 g^{2} \sum_{1 \leqslant j<k \leqslant n-1} g_{x_{j}} g_{x_{k}} g_{x_{j} x_{k}}+(n-1) g\left(1+\sum_{k=1}^{n-1} g_{x_{k}}^{2}\right)+(n-2) \frac{g_{t}^{2}}{g}
\end{aligned}
$$

Now, if $S$ is a horizontal minimal graph in $\mathbb{H}^{n} \times \mathbb{R}$, i.e. $H=0$, then the positive function $y=g\left(x_{1}, x_{2}, \ldots, x_{n-1}, t\right)$ that we call the horizontal length satisfies the equation

(3) $\mathcal{M}_{\mathcal{H}}(g)=0$

If $n=2$ we recover the horizontal minimal equation in $\mathbb{H}^{2} \times \mathbb{R}[33$, equation (2)]: 
(4) $\mathcal{M}_{\mathcal{H}}(g):=g_{x x}\left(g^{2}+g_{t}^{2}\right)+g_{t t}\left(1+g_{x}^{2}\right)-2 g_{x} g_{t} g_{x t}+g\left(1+g_{x}^{2}\right)=0$.

From now on, we focus on a 1- parameter family of elliptic equations including equation (3). Given a constant $\epsilon \in[0,1]$, we say that a positive $C^{2}$ function $g$ on a domain $\Omega$ is a solution of the $\epsilon$-horizontal minimal equation, if it satisfies the following quasilinear elliptic P.D.E:

(5)

$$
\begin{aligned}
& \mathcal{M}_{\mathcal{H} \epsilon}(g):=\sum_{k=1}^{n-1}\left[g^{2}\left(1+g_{x_{1}}^{2}+\cdots+\widehat{g_{x_{k}}^{2}}+\cdots g_{x_{n-1}}^{2}\right)+g_{t}^{2}+\frac{\epsilon}{n-1}\right] g_{x_{k} x_{k}} \\
&+\left(1+\sum_{k=1}^{n-1} g_{x_{k}}^{2}\right) g_{t t}-2 \sum_{k=1}^{n-1} g_{x_{k}} g_{t} g_{x_{k} t}-2 g^{2} \sum_{1 \leqslant j<k \leqslant n-1} g_{x_{j}} g_{x_{k}} g_{x_{j} x_{k}} \\
&+(n-1) g\left(1+\sum_{k=1}^{n-1} g_{x_{k}}^{2}\right)+(n-2) \frac{g_{t}^{2}}{g} \\
&=0
\end{aligned}
$$

Setting $a_{k k}(g, D g):=\left[g^{2}\left(1+g_{x_{1}}^{2}+\cdots+\widehat{g_{x_{k}}^{2}}+\cdots g_{x_{n-1}}^{2}\right)+g_{t}^{2}+\frac{\epsilon}{n-1}\right], k=$ $1, \ldots n-1, \quad a_{n n}(g, D g)=\left(1+\sum_{k=1}^{n-1} g_{x_{k}}^{2}\right)$ and $a_{k n}(g, D g)=-g_{x_{k}} g_{t}, k=$ $1, \ldots n-1, \quad a_{j k}(g, D g)=-g^{2} \sum_{1 \leqslant j<k \leqslant n-1} g_{x_{j}} g_{x_{k}}$, then the symmetric matrix $a_{i j}(g, D g), i, j=1, \ldots, n$ is positive, and satisfies

$$
\begin{aligned}
& \text { (6) } \sum_{i, j} a_{i j}(g, D g) \xi_{i} \xi_{j} \leqslant \operatorname{trace}\left(a_{i j}(g, D g)\right) \sum_{k=1}^{n} \xi_{k}^{2}= \\
& \left(1+\epsilon+g^{2}(n-1)+\left(g^{2}(n-2)+1\right)\left(g_{x_{1}}^{2}+\cdots+\cdots g_{x_{n-1}}^{2}\right)+(n-1) g_{t}^{2}\right)|\xi|^{2} \\
& \leqslant\left(2+g^{2}(n-1)+\left(\max \left\{1, g^{2}\right\}(n-2)+1\right)\left(g_{x_{1}}^{2}+\cdots+g_{x_{n-1}}^{2}+g_{t}^{2}\right)\right)|\xi|^{2}
\end{aligned}
$$


And

$(7)$

$$
\begin{aligned}
& \sum_{i, j} a_{i j}(g, D g) \xi_{i} \xi_{j}=\sum_{k=1}^{n-1} \xi_{k}^{2}\left(g^{2}+\frac{\epsilon}{n-1}\right)+\xi_{n}^{2}+g^{2} \sum_{1 \leqslant j<k \leqslant n-1}\left(\xi_{k} g_{x_{j}}-\xi_{j} g_{x_{k}}\right)^{2} \\
& +\sum_{k=1}^{n-1}\left(\xi_{k} g_{t}-\xi_{n} g_{x_{k}}\right)^{2} \\
& \geqslant \min \left\{1, g^{2}\right\}|\xi|^{2} \text {, } \\
& \text { where } \xi \in \mathbb{R}^{n} \backslash\{0\},|\xi|^{2}=\sum_{k=1}^{n} \xi_{k}^{2} \text {. }
\end{aligned}
$$

By invoking inequality (7), we deduce that the $\epsilon$-horizontal minimal equation (5) is a second order quasilinear elliptic equation. Of course, if $\epsilon>0$, equation (5) is strictly elliptic. If $\epsilon=0$ and if $g$ is a $C^{2}$ positive function on a bounded domain $\Omega$, continuous up to the boundary, then (5) is a strictly elliptic equation (cf. Section 3, inequality (8)).

REMARK 2.1. It is worth noticing that L. Hauswirth, H. Rosenberg and J. Spruck proved a half-space theorem for properly embedded constant constant mean curvature $1 / 2$ surfaces in $\mathbb{H}^{2} \times \mathbb{R}$, where the construction of $H=1 / 2$ horizontal graphs play a significant role in the proof [17, Theorem 1.1].

We recall now the definition of subsolution and supersolution [36], [38]. We say that a $C^{2}$ function $u: \Omega \rightarrow \mathbb{R}$ is a subsolution of equation (5) in a domain $\Omega$, if $\mathcal{M}_{\mathcal{H} \epsilon}(u) \geqslant 0$, in $\Omega$. We say that $w: \Omega \rightarrow \mathbb{R}$ is a supersolution if $\mathcal{M}_{\mathcal{H} \epsilon}(w) \leqslant 0$. It is well-known that elliptic theory ensures the following [31],[13], [32]:

Proposition 2.2 (Classical maximum principle ). Let $u: \Omega \rightarrow \mathbb{R}$ and $w: \Omega \rightarrow \mathbb{R}$ be a subsolution and a supersolution, for equation (5), respectively. Then if $u \leqslant w$ in $\Omega$ and if there is a point $p \in \Omega$ such that $u(p)=w(p)$, it follows that $u=w$ in $\Omega$.

REMARK 2.2.

(1) Is is easy to see that the Euclidean $n$-planes in $\mathbb{H}^{n} \times \mathbb{R}$ given by $y=\sum_{k=1}^{n-1} a_{k} x_{k}+b t+c ; a, b, c \in \mathbb{R}, y>0$ are positive subsolutions of equation (5). In particular the horocylinders given by $y=$ $c, c>0$, are subsolutions of (5). 
(2) Of course, the vertical geodesic n-planes $\mathcal{P}=\mathcal{P}\left(R, x_{0}\right)=$ $\left\{\left(x_{1}, \ldots, x_{n-1}, y, t\right) \in \mathbb{H}^{n} \times \mathbb{R}\right.$

$y=\sqrt{R^{2}-\left(x_{1}-a_{1}\right)^{2}-\cdots-\left(x_{n-1}-a_{n-1}\right)^{2}}, t \in(-\infty, \infty)$, $\left.R^{2}-\left(x_{1}-a_{1}\right)^{2}-\cdots-\left(x_{n-1}-a_{n-1}\right)^{2}>0\right\}$, where $R>0$ and $x_{1}, \ldots, x_{n-1} \in \mathbb{R}$, are solutions of equation (3). Moreover, it is routine to check that they are supersolutions for equation (5), as well.

(3) Minimality is invariant by a positive isometry of $\mathbb{H}^{n} \times \mathbb{R}$ given by a hyperbolic translation of $\mathbb{H}^{n} \times\{0\}$ along a geodesic $L$ [37]. Particularly, the homothety in $\mathbb{H}^{n}$ with center $\left(a_{1}, \ldots, a_{n-1}\right) \in$ $\mathbb{R}^{n-1}$, ratio $\lambda>0$, keeping $\mathbb{H}^{n}$ invariant gives rise to an isometry of the product space $\mathbb{H}^{n} \times \mathbb{R}$ given by $\left(x_{1}, \ldots, x_{n-1}, y, t\right) \mapsto$ $\left(\lambda\left[\left(x_{1}, \ldots, x_{n-1}, y\right)-\left(a_{1}, \ldots, a_{n-1}, 0\right)\right]+\left(a_{1}, \ldots, a_{n-1}, 0\right), t\right)$.

In view of this observation, we deduce that if

$y=g\left(x_{1}, \ldots, x_{n-1}, t\right)$ is a positive solution of $(3)$ on a domain $\Omega$ then $y=\lambda g\left(\frac{x_{1}}{\lambda}, \ldots, \frac{x_{n-1}}{\lambda}, t\right), \lambda>0$ is also a solution of (3) on $T_{\lambda}(\Omega)$, where $T_{\lambda}$ is the linear map given by the matrix $\left(\begin{array}{cc}\lambda I_{n-1} & 0 \\ 0 & 1\end{array}\right)$, and where $I_{n-1}$ is the $n-1 \times n-1$ identity matrix.

(4) Important model minimal hypersurfaces are the hypersurfaces invariant by hyperbolic translations [4, Theorem 3.8]. They are complete horizontal minimal graphs.

\section{UNIFORM HORIZONTAL LENGTH ESTIMATES}

In this section we infer uniform horizontal length estimates for solutions of equation (5) over bounded domains that are continuous and positive up to the boundary. In order to do that, we find minimal and constant mean curvature surfaces that will be used as barriers.

We need to define some geometric quantities that will be useful in the sequel.

DeFinition 3.1. Let $\Omega \subset \partial_{\infty} \mathbb{H}^{n} \times \mathbb{R}$ be a bounded $C^{0}$ domain with boundary $\Gamma$. Let $f \in C^{0}(\Gamma)$ be a positive function. If $n=2$, let $h(\Gamma)=\left.\max _{\Gamma} x\right|_{\Gamma}-\left.\min _{\Gamma} x\right|_{\Gamma}$ be the horizontal width of the domain $\Omega$, where $\left.x\right|_{\Gamma}$ is the restriction of the $x$ coordinate to $\Gamma$. We define

$$
R(\Omega, f):=\sqrt{\max _{\Gamma} f^{2}+\left(\frac{h(\Gamma)}{2}\right)^{2}}
$$

If $n \geqslant 3$, let $\Omega^{\pi}$ be the orthogonal projection of $\Omega$ on $\partial_{\infty} \mathbb{H}^{n} \times\{0\}$. We denote by $\operatorname{diam}\left(\Omega^{\pi}\right)$ the diameter of $\Omega^{\pi}$. 
We define $r(\Omega)$ by the radius of the smallest $n-1$ closed round disk containing $\Omega^{\pi}$, with center in $\Omega^{\pi}$. Clearly,

$\frac{\operatorname{diam}\left(\Omega^{\pi}\right)}{2} \leqslant r(\Omega)<\operatorname{diam}\left(\Omega^{\pi}\right)$.

We set

$$
R(\Omega, f):=\sqrt{\max _{\Gamma} f^{2}+r^{2}(\Omega)}
$$

We have therefore:

\section{LEMMA 3.1 (Uniform horizontal length estimates).}

Let $\Omega \subset \partial_{\infty} \mathbb{H}^{n} \times \mathbb{R}$ be a bounded $C^{0}$ domain with boundary $\Gamma$. Let $f \in C^{0}(\Gamma)$ be a positive function.

Assume that $f$ admits a positive extension $g \in C^{2}(\Omega) \cap C^{0}(\bar{\Omega})$ satisfying the $\epsilon$-horizontal minimal surface equation in $\Omega$.

Then, the following estimate holds:

$$
\min _{\Gamma} f<g<R(\Omega, f) \quad \text { in } \quad \Omega .
$$

Proof. If $n=2$ then the proof is based on the observation that the horocylinders and the vertical geodesic planes are subsolutions and supersolutions for equation (5), respectively. Let us proceed now the proof. Let $S$ be the graph of $g$, let $\left.y\right|_{S}$ be the horizontal coordinate $y$ restricted to $S$, given by the function $g$ and let $\left.y\right|_{\partial S}$ be the horizontal coordinate $y$ restricted to $\partial S$, given by the function $f$

First, we will deduce the lower bound. Let $c_{0}$ be a positive constant such that $c_{0}<\left.\min _{S} y\right|_{S}$. Consider the family of horocylinders $P_{s}, s \geqslant 0$ given by $y=c_{0}+s, s \geqslant 0$. Of course, for $s=0, S$ is contained in the mean convex side of $P_{0}$, that is, the horizontal length of $S$ satisfies $\left.y\right|_{S}>c_{0}$. Now letting $s \uparrow \infty$ we find a first point of contact of $S$ with the family $P_{s}$. By the maximum principle this first point of contact should be at a point of the boundary $\partial S$, hence the whole surface $S$ should be strictly contained in the mean convex side of the horocylinder $y=\min _{\Gamma} f$, i.e $\left.y\right|_{S \backslash \partial S}>\min _{\Gamma} f$, or equivalently $g>\min _{\Gamma} f$ in $\Omega$, as desired.

To obtain the upper bound, we argue as follows.

Consider the geodesic plane $\mathcal{P}(\Gamma)$ given by Remark $2.2(2)$, where $x_{0}=$ $x_{0}(\Gamma):=\left(\left.\max _{\Gamma} x\right|_{\Gamma}+\left.\min _{\Gamma} x\right|_{\Gamma}\right) / 2$ and $R=R(\Omega, f)=$ $\sqrt{\max _{\Gamma}\left(\left.y\right|_{\Gamma}\right)^{2}+\left(\frac{\left.\max _{\Gamma} x\right|_{\Gamma}}{2}-\frac{\left.\min _{\Gamma} x\right|_{\Gamma}}{2}\right)^{2}}$. By construction, $\partial S \subset \mathcal{P}^{+}(\Gamma):=$ $\left\{(x, y, t) \in \mathbb{H}^{2} \times \mathbb{R} ; y \leqslant \sqrt{R^{2}(\Omega, f)-\left(x-x_{0}(\Gamma)\right)^{2}}\right\}$. Consider now the family of geodesic planes $\mathcal{P}_{s}=\mathcal{P}\left(R(\Gamma)+s, x_{0}(\Gamma)\right), s \geqslant 0, \mathcal{P}_{0}=$ 
$\mathcal{P}(\Gamma)$. Of course, for $s$ big enough, we have that $S$ is contained in the same connected component of $\mathbb{H}^{2} \times \mathbb{R} \backslash \mathcal{P}_{s}(\Gamma)$ as $\partial S$, that is $S \subset$ $\left\{(x, y, t) \in \mathbb{H}^{2} \times \mathbb{R} ; y<\sqrt{(R(\Gamma)+s)^{2}-\left(x-x_{0}(\Gamma)\right)^{2}}\right\}$. Now letting $s \downarrow 0$ we cannot find a point of contact for $s>0$, by maximum principle. In contrary, we should find a first interior point of contact of $S$ with some geodesic plane of the family for some $s_{0}>0$. Since this first point of contact should be an interior point, we deduce that $S$ would be part of the geodesic plane $\mathcal{P}_{s_{0}}$. Hence, the horizontal projection of whole $S$ in $\mathbb{H}^{2} \times\{0\}$, would be contained in the geodesic (half Euclidean circle) $y^{2}+\left(x-x_{0}(\Gamma)\right)^{2}=(R(\Omega, f)+s)^{2}, y>0$, which gives a contradiction. We conclude therefore that $S \subset \mathcal{P}^{+}(\Gamma)$. Thus, we have $\left.y\right|_{S} \leqslant R(\Omega, f)$.

For the same reason we cannot find an interior point of contact when, during the movement toward the original position at $s=0$, the family reaches $\mathcal{P}(\Gamma)$. Indeed if this could happen then $S$ would be a part of the geodesic plane $\mathcal{P}(\Gamma)$, so that the horizontal projection of $\Gamma$ would be an arc of the geodesic $y^{2}+\left(x-x_{0}(\Gamma)\right)^{2}=(R(\Omega, f))^{2}, y>0$ with positive length. Since the intersection of the horizontal projection of $\Gamma$ with such geodesic consists at most of two points, we get a contradiction. Henceforth, we obtain the strictly inequality $\left.y\right|_{S \backslash \Gamma}<R(\Omega, f)$. This completes the proof of the Lemma, if $n=2$.

Now, if $n>2$ the structure of the proof is the same. Let us consider again $S=$ graph $(\mathrm{g})$. Notice that our assumption implies that $\partial S$ lies in the side of a $n$-dimensional vertical geodesic plane $S_{R(\Omega, f)} \times \mathbb{R} \subset$ $\mathbb{H}^{n} \times R$, whose asymptotic boundary contains $\Omega^{\pi}$. Where $S_{R(\Omega, f)}$ is a $n-1$ geodesic plane in $\mathbb{H}^{n}$ (Euclidean $n-1$ halfsphere) of Euclidean radius $R(\Omega, f)$.

We now accomplish the proof, as follows: We can use the $n$ dimensional horocylinders to obtain the horizontal lower length bounds for (5), in the same way as in the case $n=2$. Moreover, the $n$-dimensional vertical totally geodesic planes (Remark $2.2(2)$ ) can be employed, to obtain the desired horizontal upper bounds for (5), working similarly as in the case $n=2$.

REMARK 3.1. The proof shows that the estimate hold if we allow the solutions to be nonnegative on the boundary.

\section{UNIFORM BOUNDARY GRADIENT ESTIMATES AND MODULUS OF CONTINUITY}

In this section we build uniform barriers at any point of the boundary of a bounded convex smooth domain, for a positive smooth solution $g$ of the $\epsilon$-horizontal minimal equation $C^{1}$ up to the boundary. We obtain in fact these a priori gradient estimates in the sprit of [35], on account 
of the techniques in [13]. We also construct analytic barriers to get an uniform modulus of continuity along the boundary of a bounded convex domain, for a positive solution $g$ of the $\epsilon$-horizontal minimal equation $C^{0}$ up to the boundary.

Definition 4.1. We say that a $C^{0}$ domain $\Omega \subset \partial_{\infty} \mathbb{H}^{n} \times \mathbb{R}$ is convex if, for any $p \in \partial \Omega, \Omega$ lies in one side of some $n-1$-plane $\Pi$ of $\partial_{\infty} \mathbb{H}^{n} \times \mathbb{R}$ passing through $p$, i.e $p \in \Pi \cap \partial \Omega$ and $\Pi \cap \Omega=\emptyset$.

We need now the definition of the quantity $R(\Omega, f)$ raised in Definition 3.1 .

THEOREM 4.1 (Uniform boundary gradient estimates I). Let $\Omega \subset \partial_{\infty} \mathbb{H}^{n} \times \mathbb{R}$ be a $C^{2}$ bounded domain. Let $\varphi \in C^{2}(\bar{\Omega})$ be a positive function. Let $g \in C^{2}(\Omega) \cap C^{1}(\bar{\Omega})$ be a positive solution of the $\epsilon$-horizontal minimal equation (5) in $\Omega$, such that $g=\varphi$ on $\Gamma=\partial \Omega$. Assume that $\Omega$ is convex. Then, the following estimate holds.

$$
\max _{\Gamma}|D g| \leqslant C
$$

where $C=C\left(\min _{\Gamma} g, \max _{\Gamma} g, r(\Omega), \max _{\Omega} \varphi, \max _{\Omega}|D \varphi|, \max _{\Omega}\left|D^{2} \varphi\right|\right)$.

Proof. Let $g$ be a solution of the $\epsilon$-horizontal minimal equation as in the statement of the Theorem.

It suffices to get a priori estimates for the normal derivatives at any point $p \in \partial \Omega$. We obtain first the upper bound for the normal derivatives constructing an upper barrier.

We define

$$
\psi(d):=\frac{1}{b_{1}} \ln \left(1+\frac{e^{\left(R(\Omega, f)+\max _{\Omega} \varphi\right) b_{1}}-1}{\delta_{1}} d\right)
$$

where $b_{1}$ and $\delta_{1}$ are positive constants to be defined later.

Let $p \in \Omega$ and let $\Pi$ be the $n-1$-plane passing through $p$ as in Definition 4.1 and let $d(q)=d(q, \Pi), q \in \Omega$, where $d(q, \Pi)$ is the Euclidean distance from $q$ to $\Pi$. We define $v(q):=\psi(d(q)), q \in \Omega$. Of course, $v(p)=0$. 
Let $w=\varphi+v$ and let $\overline{\mathcal{M}}_{\mathcal{H} \epsilon}$ be the strictly elliptic operator given by (11)

$$
\begin{gathered}
\overline{\mathcal{M}}_{\mathcal{H} \epsilon}(w):=\sum_{k=1}^{n-1}\left[g^{2}\left(1+w_{x_{1}}^{2}+\cdots+\widehat{w_{x_{k}}^{2}}+\cdots w_{x_{n-1}}^{2}\right)+w_{t}^{2}+\frac{\epsilon}{n-1}\right] w_{x_{k} x_{k}} \\
+\left(1+\sum_{k=1}^{n-1} w_{x_{k}}^{2}\right) w_{t t}-2 \sum_{k=1}^{n-1} w_{x_{k}} w_{t} w_{x_{k} t}-2 g^{2} \sum_{1 \leqslant j<k \leqslant n-1} w_{x_{j}} w_{x_{k}} w_{x_{j} x_{k}} \\
+(n-1) g\left(1+\sum_{k=1}^{n-1} w_{x_{k}}^{2}\right)+(n-2) \frac{w_{t}^{2}}{g} \\
:=a_{i j}(g, D w) D_{i j} w+(n-1) g\left(1+\sum_{k=1}^{n-1} w_{x_{k}}^{2}\right)+(n-2) \frac{w_{t}^{2}}{g} \\
\text { (using summation convention) }
\end{gathered}
$$

In view of inequalities (7) and (6) we have:

$$
\begin{aligned}
& \min \left\{1, g^{2}\right\}|\xi|^{2} \leqslant a_{i j}(g, D w) \xi_{i} \xi_{j} \leqslant \\
& \quad\left(2+g^{2}(n-1)+\left(\max \left\{1, g^{2}\right\}(n-2)+1\right)|D w|^{2}\right)|\xi|^{2} \text { in } \Omega
\end{aligned}
$$

Now by invoking the horizontal length estimates (Lemma 3.1), it follows that

(13) $\min \left\{1, \min _{\Gamma} g^{2}\right\}|\xi|^{2} \leqslant a_{i j}(g, D w) \xi_{i} \xi_{j} \quad$ in $\Omega$

$$
\text { Set } \begin{aligned}
\Lambda_{1} & :=2+g^{2}(n-1)+\left(\max \left\{1, g^{2}\right\}(n-2)+1\right)|D w|^{2} \\
& =2+g^{2}(n-1)+\left(\max \left\{1, g^{2}\right\}(n-2)+1\right)|D \varphi+D v|^{2} \text { in } \Omega .
\end{aligned}
$$

We deduce the following inequalities: 
(14)

$$
\begin{gathered}
\Lambda_{1}<2+R^{2}(\Omega, f)(n-1)+\left(\max \left\{1, R^{2}(\Omega, f)\right\}(n-2)+1\right)|D \varphi+D v|^{2} \\
<2+R^{2}(\Omega, f)(n-1)+2\left(\max \left\{1, R^{2}(\Omega, f)\right\}(n-2)+1\right)\left(|D \varphi|^{2}+|D v|^{2}\right) \\
\leqslant 2+R^{2}(\Omega, f)(n-1)+2\left(\max \left\{1, R^{2}(\Omega, f)\right\}(n-2)+1\right)|D \varphi|^{2} \\
\quad+2\left(\max \left\{1, R^{2}(\Omega, f)\right\}(n-2)+1\right)|D v|^{2} \\
\leqslant\left(2+R^{2}(\Omega, f)(n-1)+2\left(\max \left\{1, R^{2}(\Omega, f)\right\}(n-2)+1\right)|D \varphi|^{2}\right)|D v|^{2} \\
\quad+2\left(\max \left\{1, R^{2}(\Omega, f)\right\}(n-2)+1\right)|D v|^{2}, \quad \text { whenever }|D v| \geqslant 1 \\
\leqslant\left(2+R^{2}(\Omega, f)(n-1)+2\left(\max \left\{1, R^{2}(\Omega, f)\right\}(n-2)+1\right)\left(|D \varphi|^{2}+1\right)\right)|D v|^{2}, \\
\quad \text { whenever } \quad|D v| \geqslant 1
\end{gathered}
$$

Set

$\alpha_{1}:=\frac{\left(2+R^{2}(\Omega, f)(n-1)+2\left(\max \left\{1, R^{2}(\Omega, f)\right\}(n-2)+1\right)\left(\max _{\Omega}|D \varphi|^{2}+1\right)\right)}{\min \left\{1, \min _{\Gamma} g^{2}\right\}}$.

By combining (13), (14) and (15) we have

(16) $\Lambda_{1}<\alpha_{1} \min \left\{1, \min _{\Gamma} g^{2}\right\}|D v|^{2}$

$$
\begin{aligned}
& <\alpha_{1} a_{i j}(g, D \varphi+D v) D_{i} v D_{j} v, \quad \text { whenever }|D v| \geqslant 1 \\
& <\alpha_{1} \mathcal{F}, \quad \text { whenever }|D v| \geqslant 1
\end{aligned}
$$

where $\mathcal{F}:=a_{i j}(g, D \varphi+D v) D_{i} v D_{j} v$.

Now set $\Lambda_{2}:=1+|D w|^{2}=1+|D \varphi+D v|^{2}$. In the same way as in proof of the inequalities (14), we deduce

(17) $\Lambda_{2}<\alpha_{2} \mathcal{F}, \quad$ whenever $|D v| \geqslant 1$

where $\alpha_{2}=\frac{3+2 \max _{\Omega}|D \varphi|^{2}}{\min \left\{1, \min _{\Gamma} g\right\}}$ 
From (11), (14), and from the definitions of $w, \mathcal{F}, \alpha_{1}$ and $\alpha_{2}$, we get

$$
\begin{aligned}
\overline{\mathcal{M}}_{\mathcal{H} \epsilon}(\varphi+v)= & a_{i j} D_{i j} v+a_{i j} D_{i j} \varphi+(n-1) g\left(1+\sum_{k=1}^{n-1}\left(\varphi_{x_{k}}+v_{x_{k}}\right)^{2}\right) \\
& +(n-2) \frac{\left(\varphi_{t}+v_{t}\right)^{2}}{g} \\
=a_{i j} \psi^{\prime \prime}(d) D_{i} d D_{j} d+a_{i j} D_{i j} \varphi+(n-1) g\left(1+\sum_{k=1}^{n-1}\left(\varphi_{x_{k}}+v_{x_{k}}\right)^{2}\right) & \\
& +(n-2) \frac{\left(\varphi_{t}+v_{t}\right)^{2}}{g}
\end{aligned}
$$

(since $d$ is linear)

$$
\begin{aligned}
& \leqslant a_{i j} \frac{\psi^{\prime \prime}(d)}{\psi^{\prime}(d)^{2}} D_{i} v D_{j} v+\Lambda_{1}\left|D^{2} \varphi\right|+(n-1) g\left(1+\sum_{k=1}^{n-1}\left(\varphi_{x_{k}}+v_{x_{k}}\right)^{2}\right) \\
& +(n-2) \frac{\left(\varphi_{t}+v_{t}\right)^{2}}{g} \\
& <a_{i j} \frac{\psi^{\prime \prime}(d)}{\psi^{\prime}(d)^{2}} D_{i} v D_{j} v+\Lambda_{1}\left|D^{2} \varphi\right| \\
& +\max \left\{(n-1) \max _{\Omega} g, \frac{n-2}{\min _{\Gamma} g}\right\}\left(1+|D \varphi+D v|^{2}\right) \\
& \leqslant-b_{1} a_{i j} D_{i} v D_{j} v+\Lambda_{1}\left|D^{2} \varphi\right| \\
& +\max \left\{(n-1) \max _{\Omega} g, \frac{n-2}{\min _{\Gamma} g}\right\}\left(1+|D \varphi+D v|^{2}\right) \\
& <-b_{1} \mathcal{F}+\left(\max _{\Omega}\left|D^{2} \varphi\right|\right) \alpha_{1} \mathcal{F}+\max \left\{(n-1) \max _{\Omega} g, \frac{n-2}{\min _{\Gamma} g}\right\} \alpha_{2} \mathcal{F} \\
& <\left(-b_{1}+\alpha\right) \mathcal{F}, \quad \text { whenever }|D v| \geqslant 1 \text {, } \\
& \text { where } \alpha:=\left(\max _{\Omega}\left|D^{2} \varphi\right|\right) \alpha_{1}+\max \left\{(n-1) \max _{\Omega} g, \frac{n-2}{\min _{\Gamma} g}\right\} \alpha_{2}
\end{aligned}
$$

Now we choose $b_{1}$ and $\delta_{1}$ such that

(1) $b_{1} \geqslant \alpha$. 
(2) $\frac{e^{\left(R(\Omega, f)+\max _{\Omega} \varphi\right) b_{1}}-1}{\delta_{1}} \geqslant b_{1} e^{\left(R(\Omega, f)+\max _{\Omega} \varphi\right) b_{1}}$.

With these choices of $b_{1}$ and $\delta_{1}$ we get that $|D v|=\psi^{\prime}(d) \geqslant 1$ if $d \leqslant \delta_{1}$.

Define $\mathcal{N}=\left\{q \in \Omega ; d(q)<\delta_{1}\right\}$. From the choices of $b_{1}$ and $\delta_{1}$ above we deduce that $w=\varphi+v$ is a positive supersolution of (11); that is, $\overline{\mathcal{M}}_{\mathcal{H} \epsilon}(\varphi+v)<0$ in $\mathcal{N}$. Observe that the linear elliptic operator $\mathcal{L}$ given by $\mathcal{L}[g-(\varphi+v)]:=\overline{\mathcal{M}}_{\mathcal{H} \epsilon}(g)-\overline{\mathcal{M}}_{\mathcal{H} \epsilon}(\varphi+v)=\mathcal{M}_{\mathcal{H} \epsilon}(g)-$ $\overline{\mathcal{M}}_{\mathcal{H} \epsilon}(\varphi+v)=-\overline{\mathcal{M}}_{\mathcal{H} \epsilon}(\varphi+v)>0$ in $\mathcal{N}$, since $g$ is a positive solution of the $\epsilon$-horizontal minimal equation, by assumption. Furthermore, $\mathcal{L}[g-(\varphi+v)]$ satisfies the Hopf maximum principle. Recall now that $g<R(\Omega, f)$, by Lemma 3.1. On $\partial \mathcal{N} \cap \Omega$ we get $g<R(\Omega, f) \leqslant \varphi+$ $R(\Omega, f)+\max _{\Omega} \varphi=\varphi+v$. On $\partial \Omega \cap \partial \mathcal{N}$ we have $g=\varphi \leqslant \varphi+v$. Hence $g \leqslant$ $\varphi+v$ in $\mathcal{N}$, by the maximum principle. Moreover, $g(p)=\varphi(p)+v(p)$. Therefore, $w=\varphi+v$ is an upper barrier (since $g(q) \leqslant w(q), q \in \mathcal{N}$ and $g(p)=w(p))$. From this, the upper bound for the normal derivatives follows. We obtain henceforth the desired boundary gradient upper bound.

To obtain the lower bound for the normal derivatives, we will construct a lower barrier. Consider $\varphi-v$. Note that $\varphi(p)-v(p)=\varphi(p)=$ $g(p)>0$. Notice that there exists a connected part $N$ of $\mathcal{N}$, containing $p$, such that $\varphi-v>0$ in $N$. We derive the following computations.

$$
\begin{aligned}
\overline{\mathcal{M}}_{\mathcal{H} \epsilon}(\varphi-v) & =-a_{i j} D_{i j} v+a_{i j} D_{i j} \varphi+(n-1) g\left(1+\sum_{i=1}^{n-1}\left(\varphi_{x_{k}}-v_{x_{k}}\right)^{2}\right) \\
& +(n-2) \frac{\left(\varphi_{t}-v_{t}\right)^{2}}{g} \\
= & b_{1} a_{i j} D_{i} v D_{j} v+a_{i j} D_{i j} \varphi+(n-1) g\left(1+\sum_{i=1}^{n-1}\left(\varphi_{x_{k}}-v_{x_{k}}\right)^{2}\right) \\
& +(n-2) \frac{\left(\varphi_{t}-v_{t}\right)^{2}}{g} \\
\geqslant & b_{1} \min \left\{1, \min _{\Gamma} g^{2}\right\}|D v|^{2}-\max _{\Omega}\left|D^{2} \varphi\right| \Lambda_{1} \\
& \operatorname{Thus} \\
& \overline{\mathcal{M}}_{\mathcal{H} \epsilon}(\varphi-v)>0, \quad \text { whenever }|D v| \geqslant 1
\end{aligned}
$$


if $b_{1}$ is chosen big enough. So with this choice of $b_{1}$, it follows that $\varphi-v$ is a subsolution of (11). Noticing that on $\partial \mathcal{N} \cap \Omega, v=\psi\left(\delta_{1}\right)=$ $\max _{\Omega} \varphi+R(\Omega, f)$, we deduce that on $\partial \mathcal{N} \cap \Omega$ we have $\varphi-v=\varphi-\max _{\Omega} \varphi-$ $R(\Omega, f)<0<g$. And on $\partial \Omega \cap \partial \mathcal{N}$, we have $\varphi-v \leqslant \varphi=g$, hence $\varphi-v \leqslant$ $g$ on $\mathcal{N}$, by the maximum principle and $g(p)=\varphi(p)-v(p)$. We thus infer the a priori lower bound for the normal derivatives. Therefore we obtain the desired a priori boundary gradient estimates. This completes the proof of the Theorem.

REMARK 4.1. For a $C^{2, \alpha}$ domain $\Omega$ for some $0<\alpha<1$ whose boundary has positive mean curvature, we can find a simpler and more geometric a priori lower bounds for the gradient. In fact, these domains satisfies a boundary slope condition and the Euclidean $n$-planes can be used as lower barriers, since they are subsolutions of the $\epsilon$-horizontal mean equation (5).

Recall now that the minimal equation in Euclidean space is given by

$$
\operatorname{div}\left(\frac{\nabla u}{W(u)}\right):=\sum_{i=1}^{n} \frac{\partial}{\partial x_{i}}\left(\frac{u_{x_{i}}}{\sqrt{1+\|\nabla u\|_{\mathbb{R}^{n}}^{2}}}\right)=0
$$

Definition 4.2. Let $\Omega \subset \mathbb{R}^{n}$ be a $C^{2, \alpha}$ bounded convex domain for some $0<\alpha<1$, with boundary $\Gamma$. Let $f \in C^{2, \alpha}(\Gamma)$. We define by $\widehat{\varphi} \in C^{2, \alpha}(\bar{\Omega})$ the unique minimal solution of the minimal equation in Euclidean space in $\Omega$, taking the prescribed boundary data $f$ on $\Gamma$ [20].

We recall that the maximum principle and the use of the Euclidean $n$-planes as barriers ensures that $\min _{\Gamma} f<\widehat{\varphi}<\max _{\Gamma} f$ in $\Omega$.

The following Lemma is well-known and we will use it in the proof of the next Lemma. We will write a proof for completeness.

LEMma 4.1. Let $\Omega \subset \mathbb{R}^{n}$ be a $C^{2, \alpha}$ bounded convex domain for some $0<\alpha<1$. Let $\Gamma=\partial \Omega$ and let $f \in C^{2, \alpha}(\Gamma)$ be a positive function.

For each $s \in[1 / 2,1]$, let $\widehat{\varphi_{s}} \in C^{2, \alpha}(\bar{\Omega})$ be the unique solution of $(20)$ taking the prescribed boundary value data $(2 s-1) f+2(1-s) \min _{\Gamma} f$ on $\Gamma$. Then there exists a constant $K$, independent of $s$, such that

$$
\max _{\bar{\Omega}}\left|D \widehat{\varphi_{s}}\right|+\max _{\bar{\Omega}}\left|D^{2} \widehat{\varphi_{s}}\right| \leqslant K
$$

Proof. For the readers convenience, we outline a proof, as follows. First, note that the Extension Lemma [13, Lemma 6.38] provides a $C^{2, \alpha}(\bar{\Omega})$ extension $\varphi_{s}$ of $(2 s-1) f+2(1-s) \min _{\Gamma} f$ such that $\max _{\bar{\Omega}}\left|\varphi_{s}\right|+$ 
$\max _{\bar{\Omega}}\left|D \varphi_{s}\right|+\max _{\bar{\Omega}}\left|D^{2} \varphi_{s}\right|$ is bounded by a constant $C$ independent of $s, s \in[1 / 2,1]$.

As we have observed before, the maximum principle yields $\min _{\Gamma} f \leqslant \widehat{\varphi_{s}} \leqslant \max _{\Gamma} f$, since this inequality occurs on the boundary. Note also that that the first eigenvalue of the matrix associated to equation (20) is 1 . Now using the extension $\varphi_{s}$, we can follow, step by step, the proof of Theorem 4.1, to ensure the bounds for the first derivatives of $\widehat{\varphi_{s}}$, independent of $s$. Then by applying the global a priori Hölder estimates of Ladyzhenskaya and Ural'tseva [21], [13, Theorem 13.7], we have global Hölder a priori estimates for the first derivatives of $\widehat{\varphi_{s}}$, independent of $s$. Finally, the global a priori Schauder estimates $\left[13\right.$, Theorem 6.6] shows that the $C^{2, \alpha}(\bar{\Omega})$ norm $\left|\widehat{\varphi_{s}}\right|_{C^{2, \alpha}(\bar{\Omega})}$, is bounded by a constant $K$, independent of $s, s \in[1 / 2,1]$. This gives the desired estimate.

Let $\ell:[0,1] \rightarrow \mathbb{R}$ be a continuous function satisfying $0 \leqslant \ell(s) \leqslant 1$, $\ell(1)=1$ and $\ell(s)=0, s \in[0,1 / 2]$. For each $s \in[0,1]$, let us now turn attention to the positive solutions of the following P.D.E:

$$
\begin{aligned}
& \text { (22) } \sum_{k=1}^{n-1}\left[g^{2}\left(1+g_{x_{1}}^{2}+\cdots+\widehat{g_{x_{k}}^{2}}+\cdots g_{x_{n-1}}^{2}\right)+g_{t}^{2}+\frac{\epsilon}{n-1}\right] g_{x_{k} x_{k}} \\
& +\left(1+\sum_{k=1}^{n-1} g_{x_{k}}^{2}\right) g_{t t}-2 \sum_{k=1}^{n-1} g_{x_{k}} g_{t} g_{x_{k} t}-2 g^{2} \sum_{1 \leqslant j<k \leqslant n-1} g_{x_{j}} g_{x_{k}} g_{x_{j} x_{k}} \\
& +\left[(n-1) g\left(1+\sum_{k=1}^{n-1} g_{x_{k}}^{2}\right)+(n-2) \frac{g_{t}^{2}}{g}\right] \ell(s) \\
& =0
\end{aligned}
$$

Note that for $s=1$, equation (22) reduces to the $\epsilon$-horizontal minimal equation.

For later purposes, we need a slight refined generalization of the boundary gradient estimates for $C^{2, \alpha}$ domains and boundary data, on account of Lemma 4.1 and the above observations.

THEOREM 4.2 (Uniform boundary gradient estimates II). Let $\Omega \subset \partial_{\infty} \mathbb{H}^{n} \times \mathbb{R}$ be a $C^{2, \alpha}$ bounded convex domain for some

$0<\alpha<1$. Let $\Gamma=\partial \Omega$ and let $f \in C^{2, \alpha}(\Gamma)$ be a positive function. For each $s \in[1 / 2,1]$, let $g_{s} \in C^{2, \alpha}(\bar{\Omega})$ be a positive solution of $(22)$ in $\Omega$, such that $g_{s}=(2 s-1) f+2(1-s) \min _{\Gamma} f$ on $\Gamma$. 
Then, for each $s \in[1 / 2,1]$ the following estimate holds.

$$
\min _{\Gamma} f \leqslant \max _{\bar{\Omega}} g_{s}<R(\Omega, f) \quad \text { on } \quad \bar{\Omega}
$$

where $R(\Omega, f)$ is given by Definition 3.1

and

$$
\max _{\Gamma}\left|D g_{s}\right| \leqslant C
$$

$$
\text { where } C=C\left(\min _{\Gamma} f, \max _{\Gamma} f, r(\Omega), K\right) \text {, }
$$

where $K$ is given by Lemma 4.1.

Proof. Let $\widehat{\varphi_{s}}$ be the unique minimal Euclidean extension of $(2 s-1) f+$ $2(1-s) \min _{\Gamma} f$ satisfying equation $(20)$.

Notice that, as in the proof of the Theorem 4.1, we have the following ingredients:

1: First, it is routine to check that $\min _{\Gamma} f \leqslant\left. g_{s}\right|_{\Gamma} \leqslant \max _{\Gamma} f$. On the other hand, observe that the Euclidean $n$-planes are subsolutions of (22). Moreover, the vertical geodesic $n$-planes (Remark $2.2(2)$ ) are still supersolutions of (22). Consequently, the length estimate inferred in Section 3 hold with exactly the same statement as in Lemma 3.1. Thus, by invoking the length estimate, it follows that $\min _{\Gamma} f<g_{s}<R(\Omega, f)$ on $\Omega$ and $\min _{\Gamma} f \leqslant g_{s}<R(\Omega, f)$ on $\bar{\Omega}$.

2: Secondly, taking into account $0 \leqslant \ell(s) \leqslant 1$, inequality (21), and inequalities (7) and (6), we are able to follow the procedure of the proof of Theorem 4.1 to obtain the desired estimate.

Noticing that in view of Lemma 4.1 and its proof, we have $K$ in the place of $\max _{\Omega}\left|D \widehat{\varphi_{s}}\right|+\max _{\Omega}\left|D^{2} \widehat{\varphi_{s}}\right|$ and $\max _{\Gamma} f$ instead of $\max _{\Omega} \widehat{\varphi_{s}}$ in the estimate.

This accomplishes the proof of the theorem.

Next, we show that a positive solution $g \in C^{2}(\Omega) \cap C^{0}(\bar{\Omega})$ of $(5)$ has a uniform modulus of continuity along a bounded convex domain $\Omega$.

Let $\Gamma \subset \partial_{\infty} \mathbb{H}^{n} \times \mathbb{R}$ be a bounded convex curve and let $f \in C^{0}(\Gamma)$. Given $\varepsilon>0$, the continuity of $f$ yields the existence of a positive constant $\delta_{0}>0$, such that $\left|f\left(p^{\prime}\right)-f(p)\right|<\varepsilon / 3$, if $\left|p^{\prime}-p\right|<\delta_{0}, p^{\prime}, p \in \Gamma$.

We now define the barriers $\varphi^{ \pm}$at $p \in \Gamma$ that we need in the next theorem. Let

$$
\varphi^{ \pm}(q):=f(p) \pm \frac{\varepsilon}{3} \pm R(\Omega, f) \frac{\ln \left(1+|q-p|^{2}\right)}{\ln \left(1+\delta_{0}^{2}\right)}, \quad q \in \Omega
$$


where $\delta_{0}=\delta_{0}(f)$ is defined in the above paragraph.

THEOREM 4.3 (Uniform modulus of continuity). Let $\Omega \subset \partial_{\infty} \mathbb{H}^{n} \times$ $\mathbb{R}$ be a $C^{0}$ bounded domain. Let $\Gamma=\partial \Omega$ and let $f \in C^{0}(\Gamma)$ be a positive function. Let $g \in C^{2}(\Omega) \cap C^{0}(\bar{\Omega})$ be a positive solution of the $\epsilon$ - horizontal minimal equation (5) in $\Omega$, such that $g=f$ on $\Gamma$. Assume that $\Omega$ is convex.

Then, it follows that given $\varepsilon>0$ there is $\delta>0$ such that if $p \in \partial \Omega$ and $q \in \Omega$, satisfy $|q-p|<\delta$, then $|g(q)-g(p)|<\varepsilon$, where $\delta=\delta\left(\varepsilon, \delta_{0}, \min _{\Gamma} g, \max _{\Gamma} g, r(\Omega), \max _{\Omega}\left|D \varphi^{ \pm}\right|, \max _{\Omega}\left|D^{2} \varphi^{ \pm}\right|\right)$.

Proof. The proof is carry out by mimicking the proof of the boundary gradient estimates, using the barriers $\varphi^{ \pm}$(instead of the function $\varphi$ ). Let $\varepsilon>0$.

Notice that $f \leqslant \varphi^{+}$on $\Gamma$ and $f \geqslant \varphi^{-}$on $\Gamma$.

We need to prove that we can find a suitable constant $\delta>0$ such that if $|q-p|<\delta, q \in \Omega, p \in \Gamma$, then both inequalities $g(q)<f(p)+\varepsilon$ and $g(q)>f(p)-\varepsilon$ hold.

To obtain the first inequality we use the barrier $w^{+}=\varphi^{+}+v^{+}$, where the function $v^{+}$is defined by $v^{+}(q):=\psi^{+}(d(q)), q \in \Omega$, where

$$
\psi^{+}(d):=\frac{1}{b_{1}} \ln \left(1+\frac{e^{R(\Omega, f) b_{1}}-1}{\delta_{1}} d\right)
$$

Indeed working exactly as in the proof of Theorem 4.1, we can deduce that $w^{+}$is a supersolution of (11) in $\mathcal{N}$, and $g \leqslant \varphi^{+}+v^{+}$in $\mathcal{N}$, where $\mathcal{N}$ is defined in the proof of Theorem 4.1. Next, we are able to choose $\delta>0$ small enough, $\delta<\delta_{0}, \delta<\delta_{1}$, such that if $|q-p|<\delta$, then $0 \leqslant \varphi^{+}(q)<f(p)+2 \varepsilon / 3$ and $0<v^{+}(q)<\varepsilon / 3$. We infer therefore that if $|q-p|<\delta$ then $g(q)<f(p)+\varepsilon$, as desired.

In order to achieve the proof of the theorem, we now define $v^{-}(q):=$ $\psi^{-}(d(q)), q \in \Omega$, where

$$
\psi^{-}(d):=\frac{1}{b_{1}} \ln \left(1+\frac{e^{\left(R(\Omega, f)+\max _{\Omega}\left|\varphi^{-}\right|\right) b_{1}}-1}{\delta_{1}} d\right)
$$

Then, we can use the barrier $w^{-}=\varphi^{-}-v^{-}$, working as before, to deduce that $w^{-}$is a subsolution of (11) in $\mathcal{N}$, and finally, to obtain that $g(q)>f(p)-\varepsilon$. This accomplishes the proof of the Theorem.

\section{UNIFORM GLOBAL GRADIENT ESTIMATES}

In this section we are able to obtain uniform a priori global gradient estimates for the $\epsilon$-horizontal minimal equation in two independent 
variables. The a priori interior gradient bound for the minimal equation in Euclidean space in two independent variables was established by $\mathrm{R}$. Finn [16]. The $n$-dimensional case was done by Bombieri, De Giorgi and Miranda [6]. Equations of minimal type were first studied by Finn [16]. He established a priori estimates for the gradient of a solution. Then H. Jenkins and J. Serrin [20] obtained curvature estimates for some kind of such equations.

In his fundamental paper L. Simon [39], derived a priori gradient estimates, for general mean curvature type equations in two variables. His results has been be applied to many mean curvature equations in two variables in several spaces [35], [10]. However, due to the geometry of the ambient space, the horizontal minimal equation does not fit the structure conditions of the equations of mean curvature type, established by L. Simon. It does not match either the structure conditions in $[13$, Theorem 5.2].

Moreover, the technique developed by L. Cafarelli, L. Nirenberg and J. Spruck in [7] to obtain a priori global gradient estimates (depending on the a priori $C^{0}$ estimates and on the a priori $C^{1}$ boundary gradient estimates), cannot be applied to the present situation. On the contrary, the method in [7] has been applied to the mean curvature equation in hyperbolic space in [27] and [3] and to a vertical constant mean curvature equation in warped product [9]. We notice that, in some cases, the a priori global $C^{1}$ estimates depends on the derivation of both a priori $C^{0}$ estimates and a priori $C^{1}$ boundary gradient estimates, see, for instance, [26] and [15].

Finally, we remark that the general a priori gradient estimates inferred by J. Spruck [40], are adapted for the vertical mean curvature equation to study many problems in several product spaces $M \times \mathbb{R}$, where $M$ is a Riemannian manifold [5], [12], [38], [8].

Next, we follow the techniques derived in [2]. We do the analysis in two independent variables, for the reasons that will be clear in the proof.

Notice that certain horizontal minimal graphs in two independent variables are Killing graphs [34]. That is they are graphs with respect to the coordinates system given by the 1-parameter group of isometries $(x, y, t) \rightarrow(\lambda x, \lambda y, t), \lambda>0$ of $\mathbb{H}^{2} \times \mathbb{R}$. This group is constituted of hyperbolic translations of the ambient space. Noticing also that by employing the translations $(\bar{x}, \bar{y}, t) \rightarrow(\lambda \bar{x}, \lambda \bar{y}, t):=(x, y, t)$, we obtain another function $y=g_{\lambda}(x, t),(x, t)$, satisfying the equation (27) for $\epsilon \lambda^{2}$ in the place of $\epsilon$ (see (45)). 
Due to the techniques employed, we are forced to make a strong constraint on the horizontal length to obtain the uniform global $C^{1}$ gradient estimates. Nevertheless, the assumption is invariant by hyperbolic translations, so it is compatible with the geometry of the ambient space.

We recall that if $n=2$ the $\epsilon$-horizontal minimal equation becomes

$$
\mathcal{M}_{\mathcal{H} \epsilon}(g)=g_{x x}\left(g^{2}+g_{t}^{2}+\epsilon\right)+g_{t t}\left(1+g_{x}^{2}\right)-2 g_{x} g_{t} g_{x t}+g\left(1+g_{x}^{2}\right)=0,
$$

THEOREM 5.1 (Uniform global gradient estimates I). Let $\Omega \subset$ $\partial_{\infty} \mathbb{H}^{2} \times \mathbb{R}$ be a $C^{1}$ bounded domain. Let $g \in C^{2, \alpha}(\Omega) \cap C^{1}(\bar{\Omega})$ be a positive solution of the $\epsilon$-horizontal minimal equation (27) in $\Omega$. Assume there exist constants $c_{1}$ and $c_{2}$, and $c_{3}$, independent of $\epsilon$, such that $0<c_{1} \leqslant g \leqslant c_{2}$ on $\bar{\Omega}$ and $|D g| \leqslant c_{3}$ on $\partial \Omega$. Assume further that $c_{2}<c_{1}+c_{1} \sqrt{\frac{\pi}{2}}$. Then there exists a constant $C$ depending only on $c_{1}, c_{2}$ and $c_{3}$ such that $|D g| \leqslant C$ on the whole $\bar{\Omega}$.

Proof. Let $g$ be a solution of the $\epsilon$-horizontal minimal equation on $\Omega$, $C^{1}$ up to the boundary, such that $0<c_{1} \leqslant g \leqslant c_{2}$ on $\bar{\Omega}$.

Assume first that $c_{1} \leqslant 1$.

By assumption, we have $c_{2}-c_{1}<c_{1} \sqrt{\frac{\pi}{2}}$. We first recall the elementary identity $\int_{0}^{+\infty} e^{-\gamma s^{2}} \mathrm{~d} s=\left(\frac{\pi}{2 \cdot 2 \gamma}\right)^{1 / 2}$. We now consider the function $\phi(u)$ given by

$$
\phi(u)=c_{1}+\int_{0}^{u} e^{-\gamma s^{2}} \mathrm{~d} s
$$

where $\gamma$ is chosen such that $c_{1}>\frac{1}{\sqrt{2 \gamma}}>\sqrt{\frac{2}{\pi}} \cdot\left(c_{2}-c_{1}\right)$. It follows that $c_{2}<c_{1}+\left(\frac{\pi}{2 \cdot 2 \gamma}\right)^{1 / 2}$.

Let $c_{12}=c_{12}\left(c_{1}, c_{2}\right)$ be a constant such that $c_{1}+\int_{0}^{c_{12}} e^{-\gamma s^{2}} \mathrm{~d} s>c_{2}$.

With this choice, we are able to write

$$
g(p)=\phi(u(p)), u \geqslant 0, p \in \bar{\Omega}
$$


for some auxiliary function $u$ satisfying $u(p)<c_{12}$ on $\bar{\Omega}$. Then, we have the inequalities

$$
e^{-\gamma c_{12}^{2}}<\phi^{\prime}(u) \leqslant 1 \quad \text { on } \bar{\Omega}
$$

and

$$
\begin{gathered}
0<-\phi^{\prime \prime}(u) \leqslant+2 \gamma c_{12} \\
\frac{-\phi^{\prime \prime \prime}(u) \phi^{\prime}(u)+\phi^{\prime \prime 2}(u)}{\phi^{\prime 2}(u)}=2 \gamma
\end{gathered}
$$

on $\Omega$.

Next differentiate (29) with respect to $x$ and $t$ to obtain

$$
\begin{gathered}
g_{x}=\phi^{\prime}(u) u_{x}, g_{t}=\phi^{\prime}(u) u_{t} \\
g_{x}^{2}+g_{t}^{2}=\phi^{\prime 2}(u)\left(u_{x}^{2}+u_{t}^{2}\right)
\end{gathered}
$$

Of course, with the aid of equation (31), we infer the following inequalities:

$$
\sqrt{u_{x}^{2}+u_{t}^{2}} \leqslant \frac{\max _{\partial \Omega} \sqrt{g_{x}^{2}+g_{t}^{2}}}{\min _{\bar{\Omega}} \phi^{\prime}(u)}, \quad \text { on } \quad \partial \Omega
$$

and

$$
\sqrt{g_{x}^{2}+g_{t}^{2}} \leqslant \sqrt{u_{x}^{2}+u_{t}^{2}}, \text { on } \bar{\Omega} .
$$

Thus, if the maximum of $D u$ on $\bar{\Omega}$ is achieved at the boundary then $\max _{\bar{\Omega}}|D u| \leqslant \frac{\max _{\partial \Omega}|D g|}{\min _{\bar{\Omega}} \phi^{\prime}(u)} \leqslant c_{3} e^{\gamma c_{12}+\gamma c_{12}^{2}}$ and we are done. Otherwise, we assume that the maximum of $D u$ is attained at an interior point $p$ of $\Omega$. Set $w(q):=\frac{u_{x}^{2}(q)+u_{t}^{2}(q)}{2}, q \in \Omega$. Thus, it suffices to infer the desired a priori estimates for the quantity $w(p)=\frac{u_{x}^{2}(p)+u_{t}^{2}(p)}{2}$. Of course, we have

$$
\begin{aligned}
w_{x}(p) & =w_{t}(p)=0 \\
\text { and } \quad u_{x}^{2} u_{x x} & =u_{t}^{2} u_{t t} \text { at } p .
\end{aligned}
$$


We remark that for the deduction of the last equation we used that we are working only with two independent variables $x, t$. This will allow us to write the second derivatives appearing in (27), in term of the first derivatives (at $p$ ). This is a crucial step to achieve the desired global estimates making use of the present method.

Hereafter, we write $\phi, \phi^{\prime}, \phi^{\prime \prime}$. Next plug the expressions (31) and its derivatives with respect to $x$ and $t$, respectively, in (27). Then rewrite the $\epsilon$-horizontal minimal equation in the form:

$$
+\frac{\phi^{2} \phi^{\prime \prime}}{\phi^{\prime}} 2 w-\frac{\phi^{\prime \prime}\left(\phi^{2}-1\right)}{\phi^{\prime}} u_{t}^{2}+\frac{\phi}{\phi^{\prime}}\left(1+2 w \phi^{2}\right)-\frac{\phi \phi^{2}}{\phi^{\prime}} u_{t}^{2}+\frac{\phi^{\prime \prime}}{\phi^{\prime}} \epsilon u_{x}^{2}=0
$$

Now note that by invoking elliptic regularity $g \in C^{3}(\Omega)$. In fact, $g$ is analytic in $\Omega$ by Morrey's regularity theorem [25]. Set $a_{11}:=$ $\phi^{2}+\phi^{\prime 2} u_{t}^{2}+\epsilon, a_{22}=1+\phi^{\prime 2} u_{x}^{2}$ and $a_{12}=a_{21}=-\phi^{\prime 2} u_{x} u_{t}$. The following identity will be useful and its verification is easy to check.

$$
\begin{gathered}
(35)\left(\phi^{2}+2 \phi^{\prime 2} w+\epsilon\right)\left(u_{x} u_{x x x}+u_{t} u_{t x x}\right)+\left(1+2 \phi^{\prime 2} w\right)\left(u_{x} u_{x t t}+u_{t} u_{t t t}\right) \\
-\phi^{\prime 2}\left(u_{x}^{2} w_{x x}+u_{t}^{2} w_{t t}+2 u_{x} u_{t} w_{x t}\right) \\
=a_{11} w_{x x}+a_{22} w_{t t}+2 a_{12} w_{x t}-\left(\phi^{2}+\epsilon+2 \phi^{\prime 2} w\right)\left(u_{x x}^{2}+u_{x t}^{2}\right)-\left(1+2 \phi^{\prime 2} w\right)\left(u_{t t}^{2}+u_{x t}^{2}\right)
\end{gathered}
$$

The conditions $a_{i j}$ is positive and $D^{2} w(p)$ is negative read

$$
\begin{gathered}
\left(\phi^{2}+2 \phi^{\prime 2} w+\epsilon\right)\left(u_{x} u_{x x x}+u_{t} u_{t x x}\right)+\left(1+2 \phi^{\prime 2} w\right)\left(u_{x} u_{x t t}+u_{t} u_{t t t}\right) \\
-\phi^{\prime 2}\left(u_{x}^{2} w_{x x}+u_{t}^{2} w_{t t}+2 u_{x} u_{t} w_{x t}\right) \\
\leqslant 0 \text { at } p
\end{gathered}
$$

Now differentiate the P.D.E (34) with respect to $x$ (respectively differentiate with respect to $t$ ) and multiply the resulting equation by $u_{x}$ (respectively multiply by $u_{t}$ ). Adding the two equations thus obtained and using (33) and (36), we infer 


$$
\begin{aligned}
& 8 \phi^{\prime} \phi^{\prime \prime} w^{2}\left(u_{x x}+u_{t t}\right)+4 \phi \phi^{\prime} w u_{x x}-2 \phi^{\prime 2} u_{t}^{2} w+6 \phi^{\prime \prime} \phi u_{x}^{2} w+2 w\left(\frac{\phi^{2}-\phi^{\prime \prime} \phi}{\phi^{\prime 2}}\right) \\
+ & 4 w^{2}\left[-\phi^{2}\left(\frac{-\phi^{\prime \prime \prime} \phi^{\prime}+\phi^{\prime \prime 2}}{\phi^{\prime 2}}\right)+\phi^{\prime 2}\right]+2 u_{t}^{2} w\left[\left(\phi^{2}-1\right)\left(\frac{-\phi^{\prime \prime \prime} \phi^{\prime}+\phi^{\prime \prime 2}}{\phi^{\prime 2}}\right)\right] \\
- & \left(\frac{-\phi^{\prime \prime \prime} \phi^{\prime}+\phi^{\prime \prime 2}}{\phi^{2}}\right) \epsilon 2 w u_{x}^{2} \geqslant 0 \text { at } p .
\end{aligned}
$$

Hence, according to the foregoing we find

$$
\begin{aligned}
& \left(8 \phi^{\prime} \phi^{\prime \prime} w^{2}\right)\left(u_{x x}+u_{t t}\right)+\left(4 \phi \phi^{\prime} w\right) u_{x x} \\
& +4 w^{2}\left[-\min \left\{\phi^{2}(u), 1\right\}\left(\frac{-\phi^{\prime \prime \prime} \phi^{\prime}+\phi^{\prime 2}}{\phi^{\prime 2}}\right)+\phi^{\prime 2}\right] \\
& \quad+\left(6 \phi^{\prime \prime} \phi u_{x}^{2} w\right)+2 w\left(\frac{\phi^{2}-\phi^{\prime \prime} \phi}{\phi^{\prime 2}}\right) \geqslant 0 \text { at } p .
\end{aligned}
$$

since $\frac{-\phi^{\prime \prime \prime} \phi^{\prime}+\phi^{\prime \prime 2}}{\phi^{2}}=2 \gamma>0$, noticing that the last term of inequality (37) is negative.

We must now estimate the quantities $u_{x x}(p)$ and $\left(u_{x x}+u_{t t}\right)(p)$ in terms of $\phi, \phi^{\prime}, \phi^{\prime \prime}$ and $w$ at $p$. Owing to (33) performing some computations we find that (34) becomes

$$
\begin{aligned}
& \text { (39) } u_{x x} \phi^{\prime}\left[\left(\phi^{2}+\epsilon+2 \phi^{\prime 2} w\right) u_{t}^{2}+\left(1+2 \phi^{\prime 2} w\right) u_{x}^{2}\right] \\
& =-u_{t}^{2} \phi^{\prime \prime} \phi^{2} u_{x}^{2}-\phi^{\prime \prime} u_{t}^{4}-\phi u_{t}^{2}-\phi^{\prime 2} \phi u_{t}^{2} u_{x}^{2}-\phi^{\prime \prime} \epsilon u_{x}^{2} u_{t}^{2} \text { at } p .
\end{aligned}
$$

and

$$
\begin{aligned}
& \left(u_{x x}+u_{t t}\right) \phi^{\prime}\left[\left(\phi^{2}+\epsilon+2 \phi^{2} w\right) u_{t}^{2}+\left(1+2 \phi^{\prime 2} w\right) u_{x}^{2}\right] \\
= & -2 \phi^{\prime \prime} \phi^{2} w u_{x}^{2}-\phi^{\prime \prime} u_{t}^{2} 2 w-2 \phi w-2 \phi \phi^{2} w u_{x}^{2}-2 \phi^{\prime \prime} \epsilon w u_{x}^{2} \text { at } p .
\end{aligned}
$$

We now perform some calculations to discover 


$$
\left(8 \phi^{\prime} \phi^{\prime \prime} w^{2}\right)(p)\left(u_{x x}+u_{t t}\right)(p) \leqslant-\frac{4 \phi \phi^{\prime \prime} w}{\phi^{\prime 2}}(p)-\left(4 \phi \phi^{\prime \prime} u_{x}^{2} w\right)(p)
$$

and

$$
\left(4 \phi \phi^{\prime} w\right)(p) u_{x x}(p) \leqslant-\frac{4 \phi^{3} \phi^{\prime \prime} w}{\phi^{2}}(p)-\frac{8 \phi \phi^{\prime \prime} w}{\phi^{2}}(p)
$$

since $\phi^{\prime}>0$ and $\phi^{\prime \prime}<0$.

Inserting (41) into (38) we then obtain

$$
\begin{aligned}
& 4 w^{2}(p)\left[-\min \left\{\phi^{2}(u(p)), 1\right\}\left(\frac{-\phi^{\prime \prime \prime} \phi^{\prime}+\phi^{\prime 2}}{\phi^{2}}\right)(p)+\phi^{\prime 2}(p)\right] \\
& +2 w(p)\left[\left(\frac{\phi^{\prime 2}-\phi^{\prime \prime} \phi}{\phi^{\prime 2}}\right)(p)-\frac{6 \phi \phi^{\prime \prime}}{\phi^{2}}(p)-\frac{2 \phi^{3} \phi^{\prime \prime}}{\phi^{\prime 2}}(p)\right] \geqslant 0
\end{aligned}
$$

Employing (30) into (42) we at last infer

$$
\begin{aligned}
& 4 w^{2}(p)\left[-2 \gamma \min \left\{c_{1}^{2}, 1\right\}+1\right] \\
& \quad+2 w(p)\left[1+\left(\left(\gamma+2 \gamma c_{12}\right) c_{2}+\left(2 \gamma+4 \gamma c_{12}\right)\left(3 c_{2}+c_{2}^{3}\right)\right) e^{2 \gamma c_{12}+2 \gamma c_{12}^{2}}\right] \geqslant 0
\end{aligned}
$$

since $c_{1}>0$.

But then,

$$
\begin{aligned}
& w^{2}(p)\left[-2 \gamma c_{1}^{2}+1\right] \\
& \quad+2 w(p)\left[1+\left(\left(\gamma+2 \gamma c_{12}\right) c_{2}+\left(2 \gamma+4 \gamma c_{12}\right)\left(3 c_{2}+c_{2}^{3}\right)\right) e^{2 \gamma c_{12}+2 \gamma c_{12}^{2}}\right] \geqslant 0
\end{aligned}
$$

since we assume that $c_{1} \leqslant 1$.

Finally, we recall that we have chosen $\gamma$, so that $2 \gamma>\frac{1}{c_{1}^{2}}$. Henceforth, in the light of (44) we derive the a priori bounds for $|D u(p)|$, if $c_{1} \leqslant 1$.

Now assume that $c_{1}>1$. Choose $\lambda<1$ such that $\lambda c_{1}<1$. Write by $\bar{y}=g(\bar{x}, t)$, the solution of $(27)$ in $\Omega$, taking coordinates $(\bar{x}, \bar{y}, t) \in$ $\mathbb{H}^{2} \times \mathbb{R}$.

By employing the translations $(\bar{x}, \bar{y}, t) \rightarrow(\lambda \bar{x}, \lambda \bar{y}, t):=(x, y, t)$, we obtain another function $y=g_{\lambda}(x, t),(x, t) \in T_{\lambda}(\Omega)$ (Remark $2.2(3)$ ), satisfying the equation (27) in $T_{\lambda}(\Omega)$ for $\epsilon \lambda^{2}$ in the place of $\epsilon$. In fact, noticing that $g_{\lambda}(x, t)=\lambda g(\bar{x}, t)=\lambda g\left(\frac{x}{\lambda}, t\right)$. It suffices to compute the relations between the first and second derivatives of $g(\bar{x}, t)$ and $g_{\lambda}(x, t)$.

We conclude therefore that $g_{\lambda}$ satisfies 


$$
\left(g_{\lambda}\right)_{x x}\left(\left(g_{\lambda}\right)^{2}+\left(g_{\lambda}\right)_{t}^{2}+\epsilon \lambda^{2}\right)+\left(g_{\lambda}\right)_{t t}\left(1+\left(g_{\lambda}\right)_{x}^{2}\right)-2\left(g_{\lambda}\right)_{x}\left(g_{\lambda}\right)_{t}\left(g_{\lambda}\right)_{x t}+\left(g_{\lambda}\right)\left(1+\left(g_{\lambda}\right)_{x}^{2}\right)=0
$$

Now the same relations between the first derivatives of $g(\bar{x}, t)$ and $g_{\lambda}(x, t)$, ensures that $\lambda^{2}\left(g_{\bar{x}}^{2}\left(\frac{x}{\lambda}, t\right)+g_{t}^{2}\left(\frac{x}{\lambda}, t\right)\right) \leqslant\left(g_{\lambda}\right)_{x}^{2}(x, t)+\left(g_{\lambda}\right)_{t}^{2}(x, t)$ and $\left(g_{\lambda}\right)_{x}^{2}(x, t)+\left(g_{\lambda}\right)_{t}^{2}(x, t) \leqslant g_{\bar{x}}^{2}\left(\frac{x}{\lambda}, t\right)+g_{t}^{2}\left(\frac{x}{\lambda}, t\right)$.

Thus

$\max _{\partial T_{\lambda}(\Omega)}\left|D g_{\lambda}\right| \leqslant \max _{\partial \Omega}|D g|$ and $\max _{\bar{\Omega}}|D g| \leqslant \frac{\frac{\max }{T_{\lambda}(\Omega)}\left|D g_{\lambda}\right|}{\lambda}$.

In view of $\lambda c_{1} \leqslant g_{\lambda} \leqslant \lambda c_{2}$, in $T_{\lambda}(\Omega)$, we can commence again (since $\lambda c_{1}<1$ ), to mimic each step of the the above procedure to obtain the desired a priori bounds, if $c_{1}>1$.

Henceforth, the a priori global gradient estimates for $|D g|$ is achieved, as desired. This completes the proof of the theorem.

In the case of a convex domain, we state the following global $C^{1}$ estimates. First, if $n=2$, equation (22) becomes (recall that $0 \leqslant$ $\ell(s) \leqslant 1, s \in[0,1], \ell(1)=1, \ell(s)=0, s \in[0,1 / 2])$.

$$
g_{x x}\left(g^{2}+g_{t}^{2}+\epsilon\right)+g_{t t}\left(1+g_{x}^{2}\right)-2 g_{x} g_{t} g_{x t}+\ell(s) g\left(1+g_{x}^{2}\right)=0,
$$

\section{THEOREM 5.2 (Uniform global gradient estimates II).}

Let $\Omega \subset \partial_{\infty} \mathbb{H}^{2} \times \mathbb{R}$ be a $C^{2, \alpha}$ bounded convex domain for some $0<\alpha<1$. Let $\Gamma=\partial \Omega$ and let $f \in C^{2, \alpha}(\Gamma)$ be a positive function. Let $\epsilon \in[0,1]$. Given $s \in[1 / 2,1]$, let $g_{s} \in C^{2, \alpha}(\bar{\Omega})$ be a positive solution of equation (46) in $\Omega$, such that $g_{s}=(2 s-1) f+2(1-s) \min _{\Gamma} f$ on $\Gamma$. Assume further that $R(\Omega, f) \leqslant \min _{\Gamma} f+\min _{\Gamma} f \sqrt{\frac{\pi}{2}}$ (where $R(\Omega, f)$ is given by Lemma 3.1).

Then, the following estimate holds.

$$
\max _{\bar{\Omega}}\left|D g_{s}\right| \leqslant C
$$

where $C=C\left(\min _{\Gamma} f, \max _{\Gamma} f, \max _{\Omega} x-\min _{\Omega} x, K\right)$.

where $K$ is given by Lemma 4.1.

Proof. We summarize the proof as follows. In view of Theorem 4.2, it is routine to check that the proof of the global gradient (Theorem 5.1) is valid for equation (46) taking $c_{2}<R(\Omega, f)$ and $c_{1}=\min _{\Gamma} f$, noticing 
that $0 \leqslant \ell(s) \leqslant 1$. Indeed, we obtain the same a priori bounds as given by equations (43) and (44).

\section{An EXISTENCE RESUlt}

Our main goal now is to prove the existence of a solution of a Dirichlet problem for the horizontal minimal equation. However, the non strictly ellipticity of the equation imposes a new insight.

Because of that, we need to consider the family of $\epsilon$-horizontal minimal equation $(27)(0 \leqslant \epsilon \leqslant 1)$ in order to solve the Dirichlet Problem for $\epsilon>0$; then, by a compactness argument, we solve the original horizontal minimal equation.

The techniques use suitable barriers and the maximum principle, combined with our a priori uniform $C^{1}$ estimates. Then, we are able to apply the Leray-Schaulder degree theory [1] and method [13], [14], to accomplish the proof.

Consider the following Dirichlet problem in two independent variables.

$$
\begin{aligned}
\mathcal{M}_{\mathcal{H} \epsilon}(g)=0 & \text { in } \quad \Omega \\
g=f & \text { on } \quad \Gamma, \quad g \in C^{2, \alpha}(\bar{\Omega})
\end{aligned}
$$

Let $R(\Omega, f)$ be the quantity defined in Definition 3.1. We have the following existence result.

TheOREM 6.1. Let $\Omega \subset \partial_{\infty} \mathbb{H}^{2} \times \mathbb{R}$ be a $C^{2, \alpha}$ bounded convex domain for some $0<\alpha<1$. Let $\Gamma=\partial \Omega$ and let $f \in C^{2, \alpha}(\Gamma)$ be a positive function.

Assume further that $R(\Omega, f) \leqslant \min _{\Gamma} f+\min _{\Gamma} f \sqrt{\frac{\pi}{2}}$.

Then, for any $\epsilon \in[0,1]$, the Dirichlet problem (48) admits a positive solution $g$.

Particularly, $f$ admits an extension $g \in C^{2, \alpha}(\bar{\Omega})$ satisfying the horizontal minimal equation (4) on $\Omega$. Furthermore, this solution is obtained as the limit in the $C^{2}$-topology of a sequence $g_{\epsilon_{n}}, 0<\epsilon_{n}<1$ satisfying (48), as $\epsilon_{n} \rightarrow 0$.

We observe that, as in Theorem 5.1, the last assumption in Theorem 6.1 is invariant by hyperbolic translations 
Proof. Suppose first $0<\epsilon \leqslant 1$. We first intend to show the existence of a solution of the Dirichlet problem (48), for $0<\epsilon \leqslant 1$.

Let $\ell(s)=2 s-1, s \in[1 / 2,1]$ and $\ell(s)=0, s \in[0,1 / 2]$. Let $h(s, p)=$ $(2 s-1) f(p)+2(1-s) \min _{\Gamma} f, s \in[1 / 2,1]$ and $h(s, p)=2 s \min _{\Gamma} f, s \in$ $[0,1 / 2]$.

Given $s \in[0,1]$ and given $g \in C^{1, \beta}(\bar{\Omega})$, consider the following family of linear Dirichlet problems:

$$
\begin{aligned}
& u_{x x}\left(g^{2}+g_{t}^{2}+\epsilon\right)+u_{t t}\left(1+g_{x}^{2}\right)-2 g_{x} g_{t} u_{x t}+\ell(s) \max \{g, 0\}\left(1+g_{x}^{2}\right)=0 \\
& \quad \text { in } \Omega, \\
& u(p)=h(s, p), \quad p \in \Gamma .
\end{aligned}
$$

Define an operator $T: C^{1, \beta}(\bar{\Omega}) \times[0,1] \rightarrow C^{2, \alpha \beta}(\bar{\Omega}), u=T(g, s)$ by the unique solution of the above problem for given $(g, s)$. Existence and uniqueness of $u$ are ensured by the extension lemma [13, Lemma 6.38] and by the classical theorem for linear strictly elliptic operator [13, Theorem 6.14]. Note that $T(g, 0)=0$ and $T(g, s)=2 s \min _{\Gamma} f$, if $s \in[0,1 / 2]$.

To ensure the existence of a solution of our Dirichlet problem in the space $C^{2, \alpha}(\bar{\Omega})$ it is suffices to check that $T(\cdot, 1)$ has a fixed point. The equation $g=T(g, s)$ reads

$$
\begin{aligned}
& g_{x x}\left(g^{2}+g_{t}^{2}+\epsilon\right)+g_{t t}\left(1+g_{x}^{2}\right)-2 g_{x} g_{t} g_{x t}+\ell(s) \max \{g, 0\}\left(1+g_{x}^{2}\right)=0 \\
& \quad \text { in } \Omega, \\
& g=h(s, \cdot) \text { on } \Gamma
\end{aligned}
$$

Then, we can apply maximum principle, comparing with Euclidean planes $y=$ cst, to ensure that a solution of the equation (49) is nonnegative, so it satisfies

$$
\begin{aligned}
& g_{x x}\left(g^{2}+g_{t}^{2}+\epsilon\right)+g_{t t}\left(1+g_{x}^{2}\right)-2 g_{x} g_{t} g_{x t}+\ell(s) g\left(1+g_{x}^{2}\right)=0 \\
& \text { in } \Omega, \\
& g=h(s, \cdot) \text { on } \Gamma .
\end{aligned}
$$

with $g \geqslant 0$ on $\Omega$.

Noticing that by applying the maximum principle we have if $s \neq 0$, $g>0$ on $\Omega$ and if $s=0, g \equiv 0$, i.e. $g$ satisfies equation (46), for $0<\epsilon \leqslant 1$. Of course, the definition of $\ell(s)$ ensures that the solution $g$ for $0 \leqslant s \leqslant 1 / 2$, is constant equal to $h(s, p)=2 s \min _{\Gamma} f$. Observe that 
the solution $g$ for $s \geqslant 1 / 2$, satisfies the inequality $g \geqslant \min _{\Gamma} f$ on $\Omega$. Now in virtue of our a priori global $C^{1}$ estimates (Theorem 5.2) and the global Hölder estimates of Ladyzhenskaya and Ural'tseva [13], we have a priori global Hölder estimates for the first derivatives. That is, there exists a constant $C$ such that $[D g]_{\Omega, \beta} \leqslant C$, for all $g$ satisfying (50). Hence, by employing the Leray-Schauder theorem [1], [13], we obtain the desired existence of a positive solution $g_{\epsilon}$ of the Dirichlet problem (48), if $0<\epsilon \leqslant 1$. Recall that the uniform a priori horizontal length estimates, given by Lemma 3.1, forces the uniform lower bound $g_{\epsilon}>\min _{\Gamma} f$ on $\Omega$, independently of $\epsilon$.

Now let $\epsilon_{n}$ be a sequence such that $\epsilon_{n} \rightarrow 0$, if $n \rightarrow \infty\left(0<\epsilon_{n} \leqslant 1\right)$ and let $g_{\epsilon_{n}}$ be a positive $C^{2, \alpha}(\bar{\Omega})$ solution of (48). Our a priori global $C^{1}$ estimates combined with the a priori Schauder global estimates, allow us to apply the Arzelà-Ascoli's theorem to obtain a subsequence $\left\{g_{\epsilon_{n_{j}}}\right\}$ that converges to a $C^{2}(\bar{\Omega})$ nonnegative function $g$ satisfying $(27)$. Clearly, $g \geqslant \min _{\Gamma} f$ on $\Omega$. Henceforth we have a solution of the Dirichlet problem (48), for $\epsilon=0$. This accomplishes the proof of the theorem.

REMARK 6.1. The geometry of the ambient space $\mathbb{H}^{2} \times \mathbb{R}$ has some very intriguing geometric phenomenon: Any catenoid (minimal surface of revolution) has vertical height less than $\pi$ and the supremum of the family is $\pi$. Eric Toubiana and the author, using the family of catenoids as suitable barriers, proved an asymptotic principle [36, Theorem 2.1] that have many consequences. In particular, it follows that there is no horizontal minimal graph given by a function $g \in C^{2}(\Omega) \cap C^{0}(\bar{\Omega})$ on a bounded strictly convex domain $\Omega$, taking zero boundary data on $\partial \Omega$. We believe that the fact that the strict convexity of the Jordan domain forbids the existence of the Dirichlet problem for (4), with zero boundary data, is a very surprising phenomenon.

Some other results in the minimal surfaces theory, make use of the behavior of the catenoid family [28], [18].

On the other hand, the following existence result is somehow a counterpart of the above remark and is a immediate consequence of Theorem 6.1. 
Corollary 6.1. Let $\Omega \subset \partial_{\infty} \mathbb{H}^{2} \times \mathbb{R}$ be a $C^{2, \alpha}$ bounded convex domain for some $0<\alpha<1$. Let $\Gamma=\partial \Omega$ and let $f \in C^{2, \alpha}(\Gamma)$ be a positive function.

Let $c_{0}$ be a constant satisfying the inequality $c_{0} \geqslant \operatorname{osc}_{\Gamma}(f)+\frac{h(\Gamma)}{2}$. Then, for any $\epsilon \in[0,1]$, there exists a solution of the $\epsilon$-horizontal minimal equation taking the boundary data $f+c_{0}$ on $\Gamma$.

\section{REFERENCES}

[1] H. Amann, "Lectures on some fixed point theorems", Monografias de Matemática, IMPA, 1974.

[2] J. Bakel'man, Hypersurfaces with given mean curvature and quasilinear elliptic equations with strong singularities, Mat. Sbornik (4) (75) 117 (1968), 561-595.

[3] L. Barbosa and R. Sa Earp, Prescribed mean curvature hypersurfaces in $\mathbb{H}^{n+1}$, with convex planar boundary, II, Sém. Th. Spect. et Geom. de Grenoble, 16 (1998), 43-79.

[4] P. Bérard and R. Sa Earp, Minimal hypersurfaces in $\mathbb{H}^{n} \times \mathbb{R}$, total curvature and index, ArXiv: 0808.38383v3.

[5] P. Bérard, R. Sa Earp, Examples of H-hypersurfaces in $\mathbb{H}^{n} \times \mathbb{R}$ and geometric applications, Mat. Contem. 34 (2008), 19-51.

[6] E. Bombieri, E. De Giorgi and M. Miranda, Una maggiorazione a priori relativa alle ipersuperfici minimali non parametriche, Arch. Rational Mech. Anal 32 (1969), 255-267.

[7] L. Caffarelli, L. Nirenberg and J. Spruck, Non-linear second order elliptic equations V. The Dirichlet problem for Weingarten hypersurfaces, Comm. on Pure and Applied Math 61 (1988), 47-70.

[8] G. Citti and C. Senni, Constant mean curvature graphs on exterior domains of the hyperbolic plane, Mathematische Zeitschrift, DOI: 10.1007/s00209-0110948-x. Published online 13 October, 2011.

[9] M. Dajczer and J. Ripoll, An extension of a theorem of Serrin to graphs in warped products, J. Geometric. Analysis (2) 15 (2005), 193-205.

[10] M. F. Elbert, B. Nelli and R. Sa Earp, Existence of vertical ends of mean curvature $1 / 2$ in $\mathbb{H}^{2} \times \mathbb{R}$, Trans. Amer. Math. Soc. (3) 364 (2012), 1179-1191.

[11] M. F. Elbert and R. Sa Earp, All solutions of the CMC-equation in $\mathbb{H}^{n} \times \mathbb{R}$ invariant by parabolic screw motion, Annali di Matematica Pura ed Applicata. DOI 10.1007/s10231-012-0268-8. Published online 1 April, 2012.

[12] J. A. Gálvez and H. Rosenberg, Minimal surfaces and harmonic diffeomorphisms from the complex plane onto certain Hadamard surfaces, Amer. J. Math. (5) 132 (2010), 1249-1273.

[13] D. Gilbarg and N.S. Trudinger, "Elliptic partial differential equations of second" order, Springer-Verlag, 2001.

[14] A. Granas and J. Dugundji, "Fixed point theory", Springer-Verlag, 2010. 
[15] E. Guio and R. Sa Earp, Existence and non-existence for a mean curvature equation in hyperbolic space, Comm. Pure Appl. An. (3) 4 (2005), 549-568. Errata: Comm. Pure Appl. An. (2) 7 (2008), 465-465.

[16] R. Finn, On equations of minimal surface type, Annals of Math. 60 (1954), 397-416.

[17] L. Hauswirth, H. Rosenberg \& J. Spruck, On Complete Mean Curvature $H=$ $\frac{1}{2}$ surfaces in $\mathbb{H}^{2} \times \mathbb{R}$, Comm. Anal. Geom. (5) 16 (2009), 989-1005.

[18] L. Hauswirth, B. Nelli, R, Sa Earp and E. Toubiana, A Schoen theorem for minimal surfaces in $\mathbb{H}^{2} \times \mathbb{R}$, Arxiv: 1111.0851v2.

[19] H. Jenkins and J. Serrin, Variational problems of minimal surface type I, Arch. Rat. Mech. Ana. 12 (1963), 185-212.

[20] H. Jenkins and J. Serrin, The Dirichlet problem for the minimal surface equation in higher dimensions, J. Reine Angew. Math. 229 (1968), 170-187.

[21] O. A. Ladyzhenskaya and N. N. Ural'tseva, "Linear and quasilinear elliptic equations" (English translation), New York, Academic Press 1968. 2nd Russian ed. 1973.

[22] L. Mazet, Magdalena Rodriguez and H. Rosenberg, The Dirichlet problem for the minimal surface equation - with possible infinite boundary data - over domains in a Riemannian surface, Proc. London Math Soc (6) 102 (2011), 985-1023 .

[23] L. Mazet, Magdalena Rodriguez and H. Rosenberg, Periodic constant mean curvature surfaces in $\mathbb{H}^{2} \times \mathbb{R}$, arXiv:1106.5900v1.

[24] C. B. Morrey, The problem of Plateau on a Riemannian manifold, Ann. Math. No. 2, 49 (1948), 807-851.

[25] C. B. Morrey, "Multiple integrals in the calculus of variations", Reprint of the 1966 Edition, Springer, 2008.

[26] B. Nelli and J. Spruck, "On the existence and uniqueness of constant mean curvature hypersurfaces in hyperbolic space", In: Geometric analysis and calculus of variations, Intern. Press, 1996, 253-266.

[27] B. Nelli and R. Sa Earp, Some properties of hypersurfaces of prescribed mean curvature in $\mathbb{H}^{n+1}$, Bull. Sci. Math. 120 (1996), 537-553.

[28] B. Nelli, R. Sa Earp, W. Santos, E. Toubiana, Uniqueness of H-surfaces in $\mathbb{H}^{2} \times \mathbb{R},|H| \leqslant 1 / 2$, with boundary one or two parallel horizontal circles, Ann. Global Anal. Geom. (4) 33 (2008), 307-321.

[29] B. Nelli and H. Rosenberg, Minimal surfaces in $\mathbb{H}^{2} \times \mathbb{R}$, Bull. Braz. Math. Soc., New Series (2) 33 (2002), 263-292; Errata: Bull. Braz. Math. Soc., New Series (4) 38 (2007), 1-4.

[30] L. Nirenberg, "Topics in nonlinear functional analysis", Courant Lecture Notes in Mathematics. American Mathematical Society, 2001.

[31] M. H. Protter and H. F. Weinberger, "Maximum principles in differential equations", Prentice-Hall, 1967.

[32] P. Pucci and J. B. Serrin, "The maximum principle" (Progress in nonlinear differential equations and their applications), Birkhäuser, 2007. 
[33] R. Sa Earp, Parabolic and Hyperbolic Screw motion in $\mathbb{H}^{2} \times \mathbb{R}$, Journ. Austra. Math. Soc 85 (2008), 113-143.

[34] R. Sa Earp, Uniqueness of minimal surfaces whose boundary is an horizontal graph and the Bernstein problems in $\mathbb{H}^{2} \times \mathbb{R}$, Mathematische Zeitschrift, DOI 10.1007/s00209-012-1001-4. Published online 22 March, 2012.

[35] R. Sa Earp and E. Toubiana, Existence and uniqueness of minimal graphs in hyperbolic space, Asian J. Math. 4 (2000), 669-694.

[36] R. Sa Earp and E. Toubiana, An asymptotic theorem for minimal surfaces and existence results for minimal graphs in $\mathbb{H}^{2} \times \mathbb{R}$, Math. Annalen (2) 342 (2008), 309-331.

[37] R. Sa Earp and E. Toubiana, "Introduction à la géométrie hyperbolique et aux surfaces de Riemann", Cassini, 2009.

[38] R. Sa Earp and E. Toubiana, Minimal graphs in $\mathbb{H}^{n} \times \mathbb{R}$ and $\mathbb{R}^{n+1}$, Ann. de l'Inst. Fourier (7) 60 (2010), 2373-2402.

[39] L. Simon, Equations of Mean Curvature Type in 2 Independent Variables, Pac. Journ. of Math. (1) 69 (1977), 245-268.

[40] J. Spruck, Interior Gradient Estimates and Existence Theorems for Constant Mean Curvature Graphs in $M^{n} \times \mathbb{R}$, Pure Appl. Math. Q. (3) part 2 (2007), 785-800.

Departamento de Matemática, Pontifícia Universidade Católica do Rio DE JANEIRO, Rio DE JANEIRO, 22453-900 RJ, BRAZIL

E-mail address: earp@mat.puc-rio.br 